\title{
A Color Vision Approach Considering Weather Conditions Based on Autoencoder Techniques Using Deep Neural Networks
}

\author{
by \\ Mohammad Mainuddin Raj \\ 16101066 \\ Samaul Haque Tasdid \\ 16101131 \\ Maliha Ahmed Nidra \\ 16301111 \\ Jobaer Noor \\ 16301210 \\ Sanjana Amin Ria \\ 17101059
}

A thesis submitted to the Department of Computer Science and Engineering in partial fulfillment of the requirements for the degree of B.Sc. in Computer Science

\footnotetext{
Department of Computer Science and Engineering

BRAC University

January 2021
}

(C) 2021. BRAC University

All rights reserved. 


\section{Declaration}

It is hereby declared that

1. The thesis submitted is our own original work while completing degree at BRAC University.

2. The thesis does not contain material previously published or written by a third party, except where this is appropriately cited through full and accurate referencing.

3. The thesis does not contain material which has been accepted, or submitted, for any other degree or diploma at a university or other institution.

4. We have acknowledged all main sources of help.

\section{Student's Full Name \& Signature:}

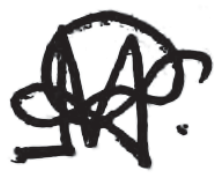

Mohammad Mainuddin Raj 16101066

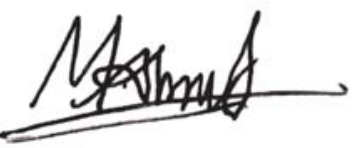

Maliha Ahmed Nidra 16301111

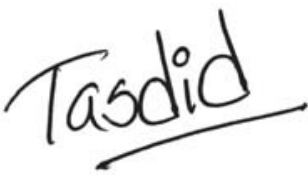

Samaul Haque Tasdid 16101131

\section{Jobaer Noor}

Jobaer Noor

16301210

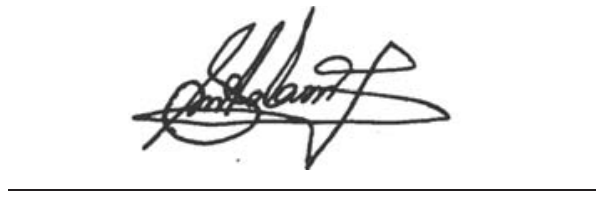

Sanjana Amin Ria

17101059 


\section{Approval}

The thesis titled "A Color Vision Approach Considering Weather Conditions Based on Autoencoder Techniques Using Deep Neural Networks" submitted by

1. Mohammad Mainuddin Raj (16101066)

2. Samaul Haque Tasdid (16101131)

3. Maliha Ahmed Nidra (16301111)

4. Jobaer Noor (16301210)

5. Sanjana Amin Ria (17101059)

Of Fall, 2020 has been accepted as satisfactory in partial fulfillment of the requirement for the degree of B.Sc. in Computer Science on January 08, 2021.

\section{Examining Committee:}

Supervisor:

(Member)

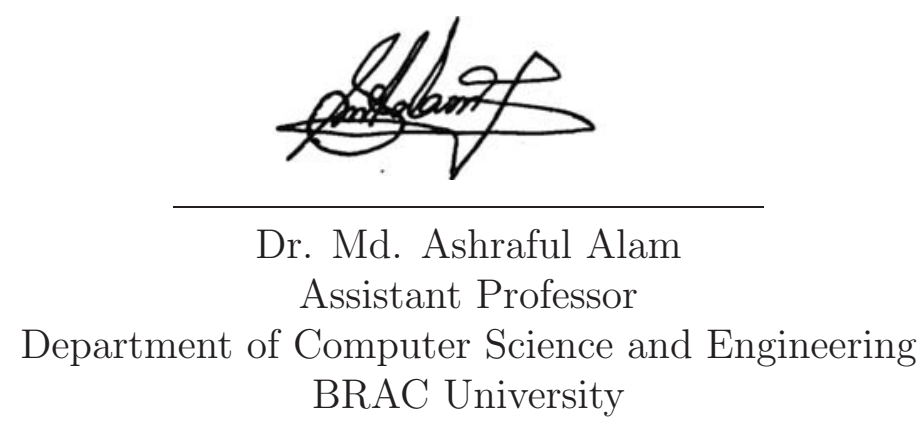

Program Coordinator:

(Member)

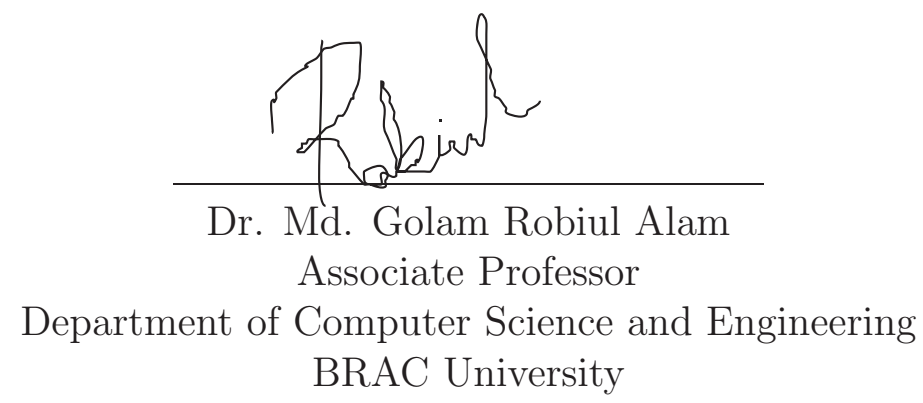

Head of Department:

(Chair)

Prof. Mahbub Majumdar

Chairperson

Dept. of Computer Science \& Engineering

Brac Universitv

Dr. Mahbubul Alam Majumdar

Professor and Dean

Department of Computer Science and Engineering BRAC University 


\section{Abstract}

Color vision approach is a riveting field of technology crucial in pioneering innovations like autonomous vehicles, autonomous drone deliveries, automated stores, robots, infrastructure and surveillance monitoring programs for security, manufacturing defect monitoring and more. When it comes to real life applications of automated machines, safety is a major concern and to ensure utmost safety the unpredictable has to be taken into consideration. We propose and demonstrate a color vision approach that allows image normalization hinged on autoencoder techniques employing deep neural networks. The model is composed of image preprocessing, encoding and decoding. The images are resized in preprocessing portion the images go through a cognitive operation where the input image becomes suitable to enter the autoencoding technique section. The autoencoder is comprised of two core components - encoder and decoder. To employ this system deep neural network is applied which generates a code of an image in the encoding process. Sequentially, the code changes over to decoding. Decoder portion decodes it and regenerates the initial image extracting it from the code of the encoder portion. It allows normalizing color images under different weather conditions such as images captured during rainy or foggy weather conditions. We devise it such that rainy and foggy images are normalized concurrently. The autoencoder is trained with numerous rainy and foggy datasets utilizing CNN. In this research, we investigate the model normalizing images in two different weather conditions - rainy and foggy conditions in real time. We used SSIM and PSNR to verify the accuracy of the model and confirm its capability reconstructing images in real time for advanced real life color vision implementations.

Keywords: Color Vision, Deep Neural Network, CNN, Autoencoder. 


\section{Acknowledgement}

The completion of this thesis would not have been possible without the constant support, help and encouragement of certain people. Praise to the Almighty for giving us the strength, patience and will to complete this project of ours and then successfully writing an entire paper on it.

We cannot express enough gratitude and we are deeply thankful to our supervisor, Dr. Md. Ashraful Alam for his patience, guidance and continuous support towards us and our thesis project. It would have been an impossible task to complete such a vastly informative and critical research paper without the help and motivation from our honorable supervisor. His critical insight to complex problems really helped us progress through our thesis at smooth pace with very few road blocks. All these have significantly helped us a lot to finish our thesis project work from home. Hence, we would like to offer our sincere appreciation for the learning opportunities provided by our supervisor.

We would also like to thank our Department of Computer Science and Engineering, BRAC University and our Faculty members who have constantly been there for us throughout our entire thesis preparation year. Their fundamental and constant guidance has played a vital role in the completion of our thesis. And last but not the least, our thesis team for working hard together and making this project a reality which was just an idea a year before. 


\section{Table of Contents}

Declaration $\quad$ i

Approval ii

Abstract $\quad$ iii

Acknowledgment iv

Table of Contents $\quad$ v

List of Figures vii

Nomenclature viii

1 Introduction $\quad 1$

1.1 Thoughts Behind the Research Topic . . . . . . . . . . . . . . . . . . 1

1.2 Motivation .......................... 2

1.3 Problem Statement . . . . . . . . . . . . . . . . 3

1.4 Research Objectives . . . . . . . . . . . . . . . . 4

1.5 Contribution . . . . . . . . . . . . . . . . 4

1.6 Thesis Organization . . . . . . . . . . . . . . . . 5

2 Literature Review $\quad 6$

3 Methodology 10

3.1 Basic Architecture of Proposed Model . . . . . . . . . . . . . . . . . . 10

3.2 Data Analysis . . . . . . . . . . . . . . . . . . 11

3.2 .1 Data Acquisition . . . . . . . . . . . . . . 11

3.2 .2 Image Pre-processing . . . . . . . . . . . . . . . . 13

3.3 Convolutional Autoencoder . . . . . . . . . . . . . . . . . . 18

3.3.1 Working Principle of Autoencoder . . . . . . . . . . . . 18

3.3.2 Autoencoder Architecture . . . . . . . . . . . . . . . 18

3.4 Mathematical Perspective . . . . . . . . . . . . . . . . . 19

4 Implementation $\quad 20$

4.1 Model Training . . . . . . . . . . . . . . . . . . . . 20

4.2 Building the Autoencoder and the Parameters Involved . . . . . . . . 21

5 Result and Analysis $\quad 29$ 
6 Conclusion 38

6.1 Limitations . . . . . . . . . . . . . . . . . . . . . 39

6.2 Future Work . . . . . . . . . . . . . . . . . . . . 39

$\begin{array}{ll}\text { Bibliography } & 42\end{array}$ 


\section{List of Figures}

3.1 Basic Architecture of Proposed Model . . . . . . . . . . . . . . . . . . 10

3.2 Rain Dataset Sample . . . . . . . . . . . . . . . . . . . . . . . . . 12

3.3 Fog Dataset Sample . . . . . . . . . . . . . . . . . . . . . . . . . . . . . . . . . . . 13

3.4 Image Resizing Process . . . . . . . . . . . . . . . . . . . . . 14

3.5 Fog Dataset (Clear image along with its 14 different density fog sample) 16

3.6 Rain Dataset (Clear image and 14 different density rain) . . . . . . . 17

3.7 The Architecture of Autoencoder . . . . . . . . . . . . . . . . . . 18

4.1 Workflow of the Model . . . . . . . . . . . . . . . . . 21

4.2 Implementation of Conv2D Maxpooling . . . . . . . . . . . . . . . . . 21

4.3 Addition of Multiple Conv2D and Maxpooling . . . . . . . . . . . . . 22

4.4 Basic Formation of Feature Map . . . . . . . . . . . . . . . . . 23

4.5 Detailed Formation of Feature Map for Three Channel (RGB) Image 24

4.6 Overview of the Feature Map . . . . . . . . . . . . . . . . . 24

4.7 Simulation of Filters Over Image Without Padding . . . . . . . . . . 25

4.8 Consequences of Adding Padding . . . . . . . . . . . . . . . 26

4.9 Graphical Representation of ReLU . . . . . . . . . . . . . . 26

5.1 Illustration of Random Test Images along with Corresponding Output 29

5.2 Reconstruction of Image Using Autoencoder . . . . . . . . . . . . . 30

5.3 Comparison of Output and It's Ground Truth (Original Image): (a) Reconstructed Image (b) Ground truth . . . . . . . . . . . . . . . . 32

5.4 Detailed Analysis of Rainy Images (a) reconstructed image, (b) ground truth, (c) differences plot in grayscale, (d) mask plot and (e) filled mask on clear image . . . . . . . . . . . . . . . . . 33

5.5 Detailed Analysis of Foggy Image (a) reconstructed image, (b) ground truth, (c) differences plot in grayscale, (d) mask plot and (e) filled mask on clear image . . . . . . . . . . . . . . . . . . 34

5.6 PSNR of Rainy Image . . . . . . . . . . . . . . . . . 36

5.7 PSNR of Foggy Image . . . . . . . . . . . . . . . . . . 37 


\section{Nomenclature}

The next list describes several symbols \& abbreviation that will be later used within the body of the document

AdaGrad Adaptive Gradient Algorithm

$A E \quad$ Autoencoder

CNN Convolutional Neural Network

Conv2D Convolution Layer

Conv2DTranspose A Convolution Operation Whose Kernel is Learnt

$D A S O M$ Denoising Autoencoder Self-Organizing Map

Kernel A Foundational Layer

MaxPooling2D A Sample-Based Discretization Process

$M S E$ Mean Squared Error

RCNN Region Based Convolutional Neural Networks

$R e L U$ Rectifier Linear Unit

RMSProp A Gradient Based Optimization Technique

$R U L$ Remaining Useful Life Prediction

SSIM Structural Similarity Index

Tensor Flow Open-source Software Library for Machine Learning

UpSampling2D A Simple Layer

YOLO An Algorithm Based on Regression 


\section{Chapter 1}

\section{Introduction}

\subsection{Thoughts Behind the Research Topic}

With the advancement of technology, the autonomous approach is rapidly being adopted by the world to solve different practical problems. These complex modern problems challenge the ability of the modern world to function autonomously and demand the world to adopt the autonomous approach of solving various problems. For example, the recent outbreak of the Coronavirus has forced the world to go into quarantine and to adapt, which broadcasts the need to espouse an online and autonomous approach to problem solving. In the fight against COVID-19, the use of machine learning has been crucial. Various machine learning and artificial intelligence based technologies are being used in healthcare industries and clinicians worldwide in order to overcome the pandemic to address the challenges throughout the pandemic [25]. Machine learning and artificial intelligence are being used in surveillance programs and for detecting criminal activities. Also, developed countries use them to monitor vehicle speeds and detect number plates to identify the vehicle owner.

Computer vision pilots computers to analyze perceptible environments using images and videos from cameras. But this has to be done in a measured and accurate way so that there remains no chance for any catastrophic failure. Usage of deep learning models enable machines to recognize and classify objects correctly and respond accordingly. Deep learning algorithms and to be specific, convolutional networks, have rapidly become a popular procedure for analysis of medical images [10]. Sophisticated computer vision applications like autonomous vehicles, autonomous drone deliveries, surveillance or critical infrastructure monitoring for security requires precise image information and analysis of considering factors like weather conditions, reflection coefficients and other factors because of which they need the application of a precise approach to eliminate the possibility of risk or failure. Our model aims exactly for that by eliminating any possible imprecise analysis. In order to do so, a lot of factors like nature, weather, light is taken into consideration while analyzing images. We mainly focus on different aspects of weather conditions while analyzing, monitor their effects on the images and remove any occurrence of anomaly and noise because of those using autoencoder techniques.

In this context we formulate this color vision approach that allows normalizing color images under different weather conditions. Here, we applied autoencoder technique 
for image normalization in adverse weather conditions like rainy and foggy weather using deep neural networks which generates and analyzes accurate real-time image information that can be used in various practical and advanced color vision/ color machine vision applications.

We have developed a model that will consider different environmental elements like rain and fog to enable composite analyzation and normalization of images. In order to do so, we employed autoencoder. Autoencoders are basically learning circuits. The core function of autoencoder is to convert inputs into outputs with as little distortion as possible [5]. We also exerted convolutional neural networks (CNN) for analyzing visual images. The structure of CNN allows it to master spatial hierarchies of attributes through backpropagation utilizing multiple building blocks deliberately, some of which are convolution layers, pooling layers, and fully connected layers, etc. [26]. For training the model we utilized open-source libraries like Keras and TensorFlow with the convenience of swift results. Keras is also an open-source neural-network library written in Python that is capable of running on top of TensorFlow and enables fast experimentation with deep neural networks. We put Conv2D convolution layer of Keras to use for training. Along with that, we have made use of filters in CNN to produce feature map in convolutional layer stride which denotes how many steps we are moving in each step in the convolution. We also used the kernel filter to extract the features from the images. The kernel is basically a matrix that moves over the input data, executes the dot product with the sub-region of input data, and retrieves the output as the matrix of dot products. We used SSIM to predict distortions and evaluate the accuracy of the model. The formidable representation power of CNN was utilized to project structural similarity maps (SSIM) which demonstrates distortion information promptly and it is done precisely: end-to-end and pixel-to-pixel [20].

\subsection{Motivation}

As the world continues to embrace automated approach to various problem solving,artificial intelligence and machine learning methods are being adopted notably. Aspiring inventions like autonomous vehicles of Tesla and Google's Waymo are being improved and pushed to limits persistently. Amazon has already deployed cashierless Amazon Go stores and Amazon Prime Air drone deliveries that implement tremendous computer vision and machine learning approach. We have also ob-served the recent launch of Spot the robot dog of Boston Dynamics which is an agile mobile robot that navigates terrain with incredible flexibility, enabling users to automate repetitive inspection activities and data capture. The possibility of this technology is colossal as it can reach hazardous places inaccessible by humans without the risk of physical injury. Computer vision is also being used in traffic monitoring and number plate detection, crime detection, social distancing monitoring and mask detection due to the recent pandemic, agriculture and farm monitoring etc. With the rapid implementation of these technologies, the safety measures have also become a huge concern. All of these implementations and their viability and accuracy depend largely on the real time weather condition. These are sensitive real-life models and cannot afford to have any defect which would lead to severe aftermath. So, we wanted to contribute on improving the accuracy of these computer vision implemen- 
tations. As Computer Science and Engineering students of BRAC University, we are offered artificial intelligence, machine learning and neural networks courses as core and elective courses that helped us get familiar with the topics. This way our motives were harmonized and it motivated us to conduct the research. In addition, the potential for this technology in the future is apparent and there is also scope for collaborations. We could integrate our model with other models that consider correlating factors and create a functional model. This would enhance the technology remarkably and make it more resilient.

\subsection{Problem Statement}

There can be a lot of hindrances in the way of a successful image analysis and weather condition is among them. A model may operate error-free on a normal sunny day, but its ability to operate in a more adverse weather condition should also be taken into consideration. We are envisioning real life practical applications where a simple error can lead to catastrophic disruption. For example, we can mention self-driving autonomous vehicles which are already being manufactured and sold by companies like Tesla and Waymo in developed countries. If an autonomous vehicle were to detect objects inaccurately due to raindrops or snowflakes, it may lead to accidents which will disrupt the whole purpose of this technology. Again, if a critical infrastructure monitoring for security was to not analyze objects properly, it can lead to a security breach which contradicts the whole purpose of using an autonomous system. So, we took all the aspects of possible flaws into consideration. Adverse weather conditions are a huge problem in analyzing images. If the image is captured on a normal sunny day, it will be easier to analyze than an image taken on a rainy or foggy day. If the image is of a rainy or foggy day, the input image will be unclear and noisy because of rain and fog and there is also insufficiency of light on a rainy or foggy day. In these cases, we have to eliminate the noise created by rain or fog or deficient light in order to normalize the image for accurate operation of the model. It has to be trained with challenging images and also has to be equipped with proper technology in order to overcome these obstacles. Various models are currently being used for object detection like RCNN, YOLO V2/V3 but they are not recommended for adverse weather conditions. In order to mitigate this kind of problem, there has been use of night vision cameras, thermal imaging and color histogram features. We are certain that our model is going to contribute tremendously in solving this problem. We approached it in a methodical way by making measured use of autoencoder and CNN algorithms for real time image processing and object detection. If we used any generic algorithm for object detection, the model could provide incorrect results as the image pattern could get transfigured due to raindrops or fog etc. It can be hazardous as the model might detect the object inaccurately from its knowledge which might lead it to make a wrong decision. The application sectors of our model have crucial real life and real-time use cases and that is why there was no room for any blunder or else it would lead to dire consequences. Conventional vision systems are generally intended to work in clear weather. But external vision systems should contain methods to generate output in adverse weather conditions. Also, the atmosphere influences the light energy before it reaches the witness [3]. In the article [3], the authors use atmospheric scattering models to make vision systems robust in adverse weather. 
We have put in our effort to contribute to the sector with our model with a view to enrich it and have significant impact on the development. Since the regular models with generic algorithms cannot ensure adequate security, they have to be improved. With the motivation to improve it, we used autoencoder technique and CNN algorithms to eliminate that possible room for error.

We made the measured steps of our system like pre-processing, cognitive operation, autoencoding technique section, code conversion and decoding work accurately. The model then removes the noise from the image and reconstructs it to get precise normalized images that is able to detect objects with great accuracy. Accurate object detection despite the weather conditions means the probability of happening any accident or security breach diminishes significantly which should aspire interest to use our model. To ensure the reliability of our system and to make the world a safer and functional place with autonomous applications was the main motivation to drive us to truly implement the model.

\subsection{Research Objectives}

An image captured in different weather conditions like rainy or foggy weather is disrupted by having raindrops or haziness, resulting in the implementation of that particular image to be inconsistent and inaccurate. Thus, by using our model, that particular image is converted to an image as if it was captured in normal daylight condition by normalization, getting rid of the impact of unwanted weather conditions. Our main objective was to identify objects through image processing in different weather conditions and in that track, our brief procedures were-

(i) Preparing a dataset of images in various weather conditions.

(ii) Training the model by utilizing the data.

(iii) Reconstructing images through deep neural networks.

(iv) Detecting abnormality and noise in images by training the model with numerous images to make it persistent in identifying and differentiating objects.

(v) Monitoring the performance of the model.

(vi) And thus, render clear images for detecting objects accurately despite the weather condition.

\subsection{Contribution}

There have been quite a few works in the sector of color vision or computer vision sector that utilizes neural networks or autoencoder. But there has not been much work on models that take weather conditions into consideration or have real time implementations. For example, there have been researches on denoising autoencoder self-organizing map (DASOM) that incorporates the latter into a hierarchically arranged composite version where a grid of topologically ordered neurons forge the front-end portion [16]. Also, there have been proposed models like updated deep learning-based prediction method for using deep autoencoders and deep neural networks for bearing RUL [17] and prediction of transit networks for short-term traffic congestion with a view to solve the traffic problem [22]. Models utilizing distortions in recognizing noisy images with neural networks have also been introduced [8]. These models do not take any environmental factors into consideration which 
may lead them to glitch in certain weather conditions.

In comparison, our model not only detects objects from noisy images due to rain or fog but also does it in real time which makes our model more reliable and applicable to more sophisticated and frontline executions. Our model takes rain or fog disrupted images, removes the anomaly and detects the precise object to eliminate any underlying hazard. Hence, our model we believe will triumph over any other models in perilous weather conditions thus corroborating its reliability and efficiency.

\subsection{Thesis Organization}

In this era where people are constantly working towards technological perfection and improvement, our paper is working out a solution towards major hindrances caused by noisy images using an autonomous approach. We have tried to explain our approach in details in total six chapters which we further divided to twenty-three parts in this paper. In the first part of our thesis, Chapter 1, we have mentioned how over the years Machine Learning and Artificial Intelligence has been a vehicle towards progress and tackling various complex problems. We have also talked about its background and history of uses in details before proposing our goal which we wish to achieve through autonomous approach of developing a model that will consider different environmental elements concurrently to enable composite analysis and normalization of images. We have further talked about our motivation in the sub sections i.e., our will to contribute to aid on improving the accuracy of computer vision implementations. We then stated the problem we are attending to, our objective for this research and our contributions. Moving on to Chapter 2, here we have discussed in details all that we got to learn from the papers we have researched and studied for our thesis, where we talked about their approach, their methodology, their limitations (if any) and how we can or cannot relate to their paper. In this chapter we can find a total of 10 papers chosen from many more that we thought were relevant and reviewed those in details. Next, we have discussed our proposed model in details in Chapter 3, i.e., our methodology. There we have showcased our proposed model, the work flow and the architecture of our autoencoder. We explained our reason for choosing Convolutional Neural Network, i.e., higher accuracy. The working principle of autoencoding is also explained thoroughly, the model training and the three major components that the autoencoder is comprised of. In this chapter we come across how we acquired and processed our data for our autoencoder to train. Concluding chapter 3, we move onto Chapter 4, where we define our algorithm further for every step of our model and showcase and discuss the results of those algorithms. Here in the subsections, we can find the model training, the algorithms used for building the encoder and the parameters that have been used. Finally, in Chapter 5, we can find the result of our paper before we conclude in the next chapter (Chapter 6). 


\section{Chapter 2}

\section{Literature Review}

In today's world, image processing is becoming a vital part of computer science. Computer vision is becoming the most important factor in this digital universe. In order to interconnect material world with technological, it plays a significant role and is one of the most prominent method. We are working on a paper where we can autoencode our image to remove specific weather factor and create an abstract free image. Our approach is based on autoencoder technique using Deep Neural Network and taking account of weather conditions. There are many research papers that have been published on image processing. Also, there are many papers regarding the noise removal issues. But in our paper, we try to solve specific weather factor issues like rain and fog. We found many research papers related to the topic. And we try to understand their methodology and find their limitations. We found a paper titled "UNSCARF-a color vision system for the detection of unstructured roads" [1], where the authors try to build a color vision system to detect road for an intelligent mobile robot. Here they used a UNSCARF algorithm. This algorithm collects image and checks for similar pixel in the color image from its training dataset. First it reduces the color image size and then removes the noise and converts the input color image in required size, then it matches it with its own data. This system is very slow and costly. Besides it cannot always generate the actual proper images for the robot. In our paper we will be using a system where we used autoencoder techniques which is far more efficient and effective. In our studies we found another paper titled "LLNet: A deep autoencoder approach to natural low-light image enhancement" [11]. Here the authors used deep autoencoder techniques for LLNet or low light net, where they tried denoising it using BM3D, K-SVD and non-linear filters. Then they used various deep learning techniques for LLNet. LLNet used 3 DA layers. And these 3 layers becomes bottleneck layer. They trained their dataset with patches extracted from their standard test images. They used two performance metric PSNR and SSIM to evaluate the proposed framework's performance. We used similar method like autoencoder and bottleneck in our system. Beside that we are also going to use our test images to train our dataset. Their [11] paper purely focused on low light image enhancement and our system is focused on removing weather condition from image, so our output and other methodology is different from their [11] system. We came across another paper titled "A joint deep neural networks-based method for single nighttime rainy image enhancement". In this paper [19] the authors try to build a new system which will derain image in low-illumination situations. They used a symmetric contextual autoencoder neural network to enhance a single night 
time image.

They also [19] used a retinex theory to decompose image. On our paper we used deep autoencoder to reconstruct rain and fog free images. In our paper we mainly focused on overall weather factors in any image. We try to build a model to point out the rain and fog and then try to remove them from our test image. As in theirs [19], they are specifically removing only rain from single low-illuminating image; hence our way of work and methodology is different from theirs. We found another paper named "Clearing the Skies: A deep network architecture for single-image rain removal" [9]. In this paper [9] authors implement a new deep convolutional neural network system called DrainNet to remove rain streaks from an image. With the help of the DrainNet system they [9] also try to improve the visual quality of the image. They train their model with high pass detail layer. They [9] also have given different parameter settings for Kernel size, Network width and Network depth. As we are trying to work with multiple weather factors, so we cannot directly use DrainNet. But instead, we will be using convolutional neural network and autoencoder. We also try to enhance our output image quality using autoencoder. Beside that we will also be using different parameters for the training of our model. We came across another paper related to our paper, this paper is named "Image recognition with deep neural networks in presence of noise - Dealing with and taking advantage of distortions" [8]. This paper is mainly focused on experimental examination of the influence of different types of noise on the convolutional neural network. This paper also come up with a denoiser using deep neural network. They evaluated two possible strategies of dealing with noise; one is training data augmentation and another is denoising prior to classification. They also did classification performance under various noise settings and different noise model. We studied this paper [8] thoroughly for understanding denoising and deep neural network. Since in our paper we will be using a deep neural network model. Another paper we found named "Medical image denoising using convolutional denoising autoencoders" [7]. In this paper, authors used a convolutional denoising autoencoder to remove noise from various Medical imaging including X-rays, Magnetic Resonance Imaging (MRI), Computer Tomography (CT), ultrasound etc. They [7] used two datasets, mini-MIAS database of mammograms (MMM) and a dental radiography database (DX). They sized all the images from the dataset and trained their model. We studied this paper [7] to find out their methodology which helped us to understand how autoencoder works in different situations, as their [7] work mainly focuses on medical imaging and we work on normal images. The next paper we researched was "Image Denoising and Inpainting with Deep Neural Networks" [6]. In this very well-descriptive paper, the authors have tried and wielded great results in solving a low vision problem by denoising and inpainting using deep neural network. This paper [6] had a similar goal to ours, except here, the author worked on denoising more generalized conditions, whereas we tried to work on a more targeted problem of denoising specific weather conditions. To prepare their [6] data, they first tried to corrupt their images with additive white Gaussian noise of various standard deviations using which they have trained their DA with, whereas for our data, we have used image augmentation method. They [6] have also worked on inpainting the images to restore their data images as close to its original form. To serve this purpose, in this paper [6], they have used Denoising autoencoder (DA) and Stacked Sparse Denoising Autoencoders 
and compared their results with KSVD and BLS-GSM. The authors [6] mentioned, the limitations they faced during their project was due to SSDA as it strongly relies on supervised training, hence, their proposed model of blind inpainting can only remove the noise patterns it has seen earlier during its training in the training data, which would be a massive disadvantage in our case as weather condition images would rarely be in a similar pattern and hence this methodology would not work for our project. From this paper [6] we gathered knowledge about DA and SSDA methodology which helped us in further deciding our preferred method which will best serve our goal.

After that we would like to discuss the paper "Classification of Massive Noisy Image Using Autoencoders and Convolutional Neural Network". Here we get to study another paper that is working with Convolutional Neural networks. In this paper, the authors [12] have tried to reconstruct native images from noisy input images by creating a hybrid model. We could greatly relate to this paper [12] and studied the paper rigorously to muster all the knowledge we can gain from it. Here, the authors [12] have tried to denoise the images using Denoising autoencoder, Convolutional denoising autoencoder and also a hybrid of both. They tried total three hybrid methods: In the first one they trained a DAE and a CDAE to reconstruct their native images from noisy images. In their second experiment, they tried to construct a CDAE-CNN Architecture using CDAE followed by a CNN and lastly, they constructed DAE-CDAE-CNN architecture combining DAE for denoising input images, CDAE for representing the reconstructed images better and a CNN classifier for classification of images. They [12] then compared their results and declared that the final approach provides better result. The purpose of their paper [12] is to develop a supervised classifier which will be able to show better performance in classifying noisy image data, whereas ours is an unsupervised system, also we tried to keep our system simple yet learning and performing its task effectively.

While researching various papers about denoising images, we also came across a paper named "Image Restoration using Autoencoding Priors" [13]. The authors of this paper worked on restoring, not only noisy images but also blurred images. They used denoising autoencoders (DAE) that they have trained using Gaussian noise but unlike our autoencoder or most neural autoencoders, their autoencoder does not have a bottleneck section for the code, as they mentioned in their paper [13] that the latent space is not required for DAE training and also, they do not seek its requirement for their application. For their paper [13], they have also performed the process, Super resolution to not only achieve a clean image but also an image of higher resolution as the target of their paper was basically image restoration whereas we have highly targeted denoising our images to achieve a clean image without any traces of disturbance caused by weather conditions.

"Improved Denoising Autoencoders for Image Denoising" [18] is the next paper that we came across. The authors, in this paper has talked about how Denoising autoencoder outperforms several other conventional denoising algorithms including the original Denoising Autoencoders, total variation (TV) minimization, BM3D and non-local mean (NLM) algorithms. To prove their claim, the authors use Denoising autoencoders and they propose an improved training loss function for Denoising 
Autoencoders that are based on Method noise and entropy maximization principle, including residual statistics as constraint conditions and lastly to verify the claim, they compared their results with other denoising algorithms. Even though this paper was very intriguing, the purpose of this paper doesn't quite match ours, but nonetheless, this paper helped us judge the compared autoencoding techniques through the result comparisons that we see in this paper [18] and also allowed us to understand and study their development and evaluation of a loss function construction method for denoising autoencoders.

The last paper we are going to discuss is named "Noise Removal from Color Images" [2]. Here, Zheng and Gauch[2] talked about Color image analysis, chromaticitypreserving median filter, color perception, monochromatic like processing, vector signal processing and mean type filtering. Although this paper was written long ago and the technology developed a long way after their paper [2] was published, but here they tried to describe the very basic buildup of image processing, filtering and vector modeling to remove noise. Even though in our paper we will be using more modern ways but from this paper [2] we tried to understand the basic methodology. 


\section{Chapter 3}

\section{Methodology}

As mentioned earlier, the primary goal of this paper is to develop a system that normalizes color images under different weather conditions such as rain and fog. For this, we proposed autoencoder technique for normalizing the images under weather conditions like rainy and foggy weather using deep neural networks. Hence, we developed a model that considers rainy and foggy conditions concurrently, analyses the input data images and provides a normalized image.

\subsection{Basic Architecture of Proposed Model}

The basic architecture of the proposed model is illustrated below: -

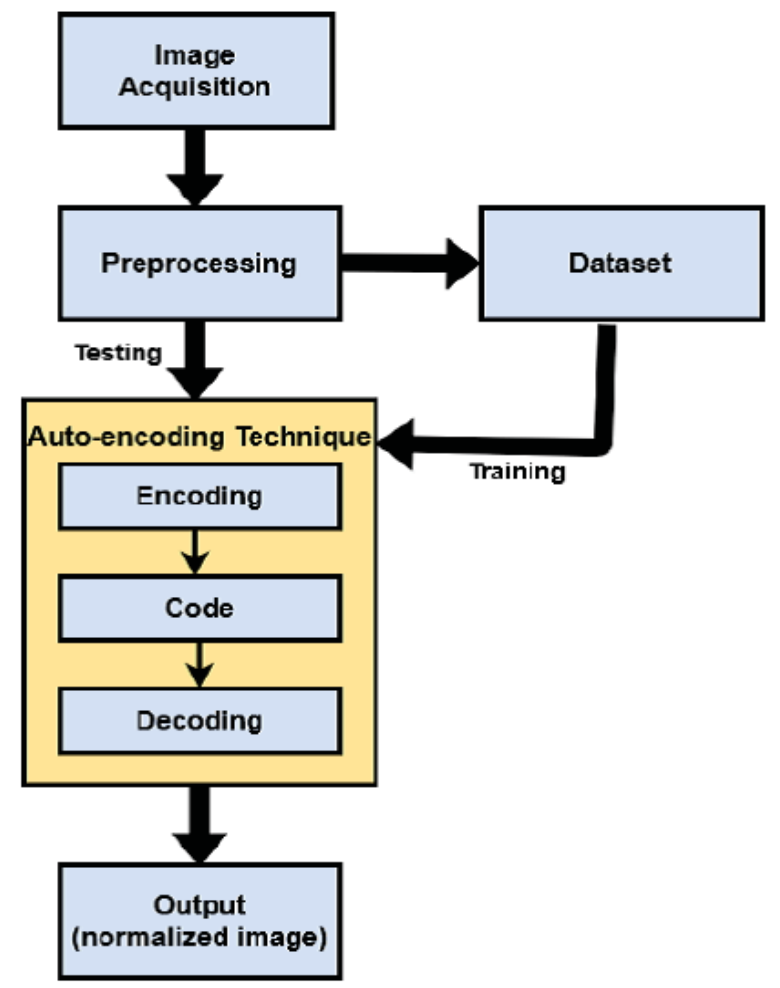

Figure 3.1: Basic Architecture of Proposed Model 
In Figure 3.1, the model we proposed has been divided into different steps for successfully generating a clear normalized image by performing image acquisition followed by image pre-processing and finally the autoencoding technique is implied in order to get our result. On the very first step, in image acquisition, image data is to be captured or collected and propagated to the second step, Image pre-processing. This is where the initial raw data is transformed into a required format which we have used as our dataset. Using this collected dataset, we move onto our third step, the autoencoding technique, where the autoencoder is trained. After all of these steps are performed successfully and the autoencoder has been trained effectively, the model is then able to take the pre-processed images and directly normalize them through the trained autoencoder.

\subsection{Data Analysis}

Data analysis is defined as a process of cleaning, transforming, and modelling data to discover useful information, eliminating unnecessary information and features from the data to retrieve the most suitable and readable form we can accept as input.

As the purpose of our autoencoder is to take an image that contains rain or fog and deliver a clean image by removing the traces of rain or fog from the images, hence we will be needing images containing rain and fog for training and testing our autoencoder. To train our model, we need large number of dataset but due to certain limitations we could not manage to collect enough images and hence we had to choose secondary dataset, along with our captured images.

\subsubsection{Data Acquisition}

For our thesis we tried to build our own dataset since we are going through a COVID19 pandemic time, we had to maintain all the health safety protocols. Besides that, as mentioned earlier, during our research period we have not come across much suitable weather situations in our country's weather to gather adequate amount of rain and fog image dataset. For these particular reasons we had to take some help from other sources as well and we tried to gather adequate amount of data by finding more dataset that is best suitable for our thesis. In order to find these dataset, we kept two things in mind, the first thing is the dataset quality and the second thing is the dataset's usability. In dataset quality we look for best possible dataset where images are in good condition and images have clear conditions. We have come across various dataset where images were pixelated, some had dataset images that were blurry, and furthermore, some dataset had images that has random regulations and quality which is not very suitable for our thesis. The second thing we looked for was dataset's usability. Some dataset that we found had usability as low as 2, some had usability of 2.5. For that reason, we tried to find dataset which has high usability rate. After our search, we actually managed to find two sets of dataset of rainy and fogy images that satisfies both of our conditions, i.e., the dataset have clear and good condition image with high usability rate and hence, we finally were able to use those in our thesis. Few of the rain dataset that were added were taken from the dataset of Wang, Tianyu and Yang, Xin and Xu, Ke and Chen, Shaozhe 
and Zhang, Qiang and Lau, Rynson W.H [21] and for haze dataset, we took help from the dataset of Ashwath,B [23]. These dataset have a high usability rate, higher than 9 and used synthesized data to add rain and fog. So, we took to these dataset to increase our training dataset by merging with ours that we personally gathered, some by capturing naturally occurring rain and fog and some by gathering raw plain images without rain or fog and adding synthesized rain droplets later.
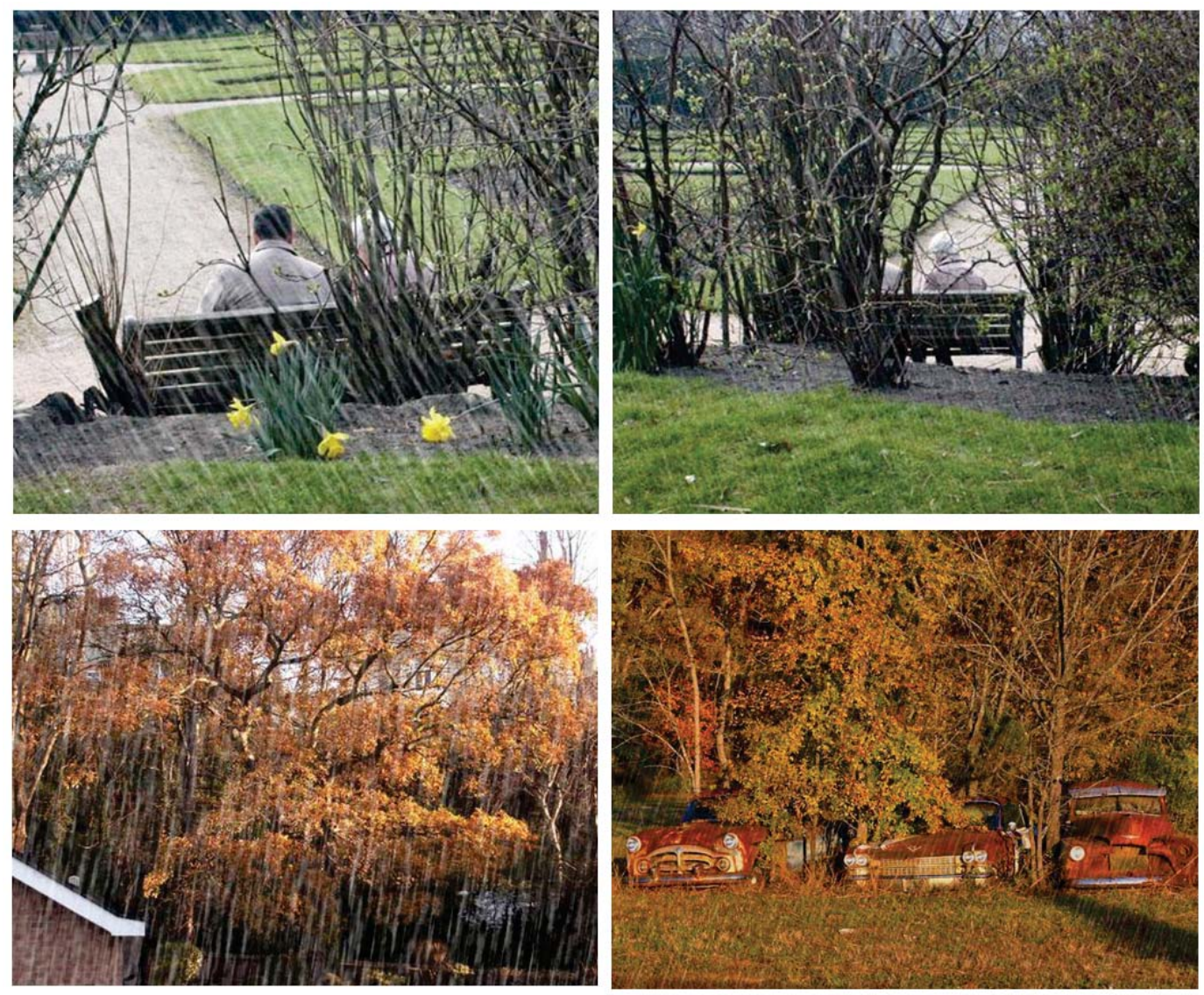

Figure 3.2: Rain Dataset Sample 

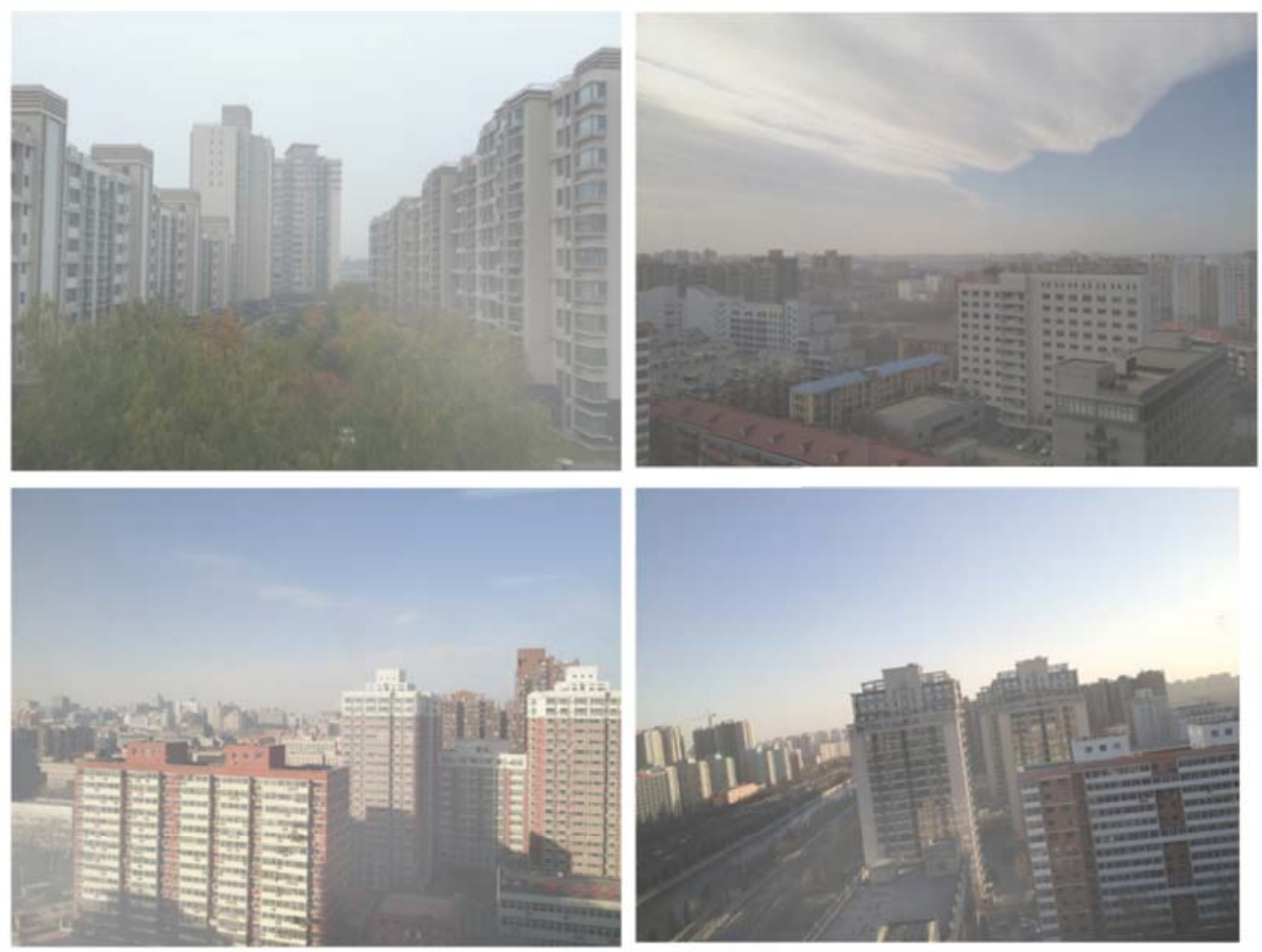

Figure 3.3: Fog Dataset Sample

\subsubsection{Image Pre-processing}

Image processing is the use of computer algorithms to perform image processing on digital images. Digital image pre-processing can be done using a wide range of algorithms that are applied to the input data to prepare the image for further machine usage- the aim of digital image processing is to improve the image data (features) by suppressing unwanted distortions and/or enhancement of some of the more important image features that will be needed in order for our Autoencoder model to be benefitted by this improved data, so that it can further work on it.

An image is nothing more than a two-dimensional array of numbers (or pixels) ranging between 0 and 255. It is defined by the mathematical function $f(x, y)$ Where $x$ and $y$ are the two co-ordinates horizontally and vertically.

The value of $f(x, y)$ at any point is giving the pixel value at that point of an image.

The color images are normally represented using 3 channels of RGB: Red, Green and Blue. When we use RGB image as input to CNN, the depth of filter (or kernel), it will always have to be same as the depth of image. For RGB this depth is 3 . Hence, if the input image $M * M * 3$, the filter will have to be $N * N * 3$ ( $N$ will be the height and width of filter here). Hence, we have a filter with 3 two dimensional matrices where separately in every single plane each of the two-dimensional matrices has been combined with a small portion of the provided input image and these results from each of the plane are added to generate a single output value in the feature map.

Matplotlib for plotting vast variety of graphs, starting from histograms to line plots 
to heat plots. We can use Pylab feature in ipython notebook (ipython notebook - pylab $=$ inline) to use these plotting features inline. If we ignore the inline option, then pylab converts ipython environment to an environment, very similar to Matlab. We can also use Latex commands to add math to our plot.

We have been loading a single image and we've looked into the data formats specific to that image and extracted mode and size of the image. The mode of an image defines the type and depth of a pixel in the image. The size of an image is (height * width).

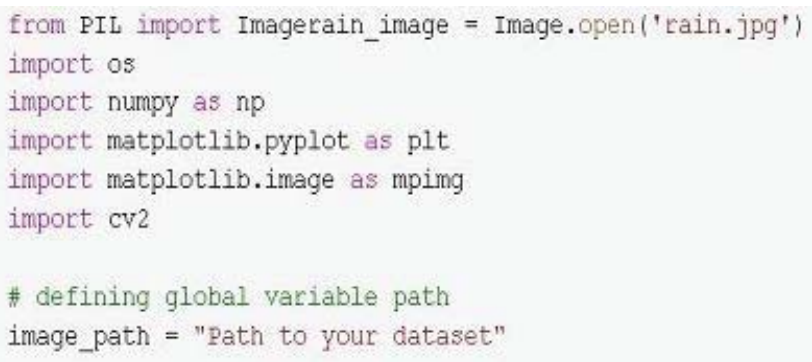

Figure 3.4: Image Resizing Process

After that is done, we then resized all the images to $256^{*} 256$ pixel size, as the images that are collected and fed to our encoder varied in sizes, therefore, we needed to establish a fixed base size for all the images.

In the next step, in order to visualize the change, we created two functions that displayed the images, the first being the one to display one image and the second for two images. After that, we then created a function called processing that will just receive the images as a parameter and work on it.

For the system to be more accurate we also considered the fact that rain droplets usually don't have the same angle and that the angle varies considering both terminal vertical speed of raindrops $(V T)$ and the average horizontal wind speed near the ground $(V S)$. Rain streaks are generally at an angle $(\theta)$ compared to the vertical between, $0($ ver $)<\theta(\mathrm{rad})<\pi / 2$.

Hence, under the condition of assuming the angle of the rainfall to be constant within each time interval, we can calculate the angle using the formula:

$$
\theta=(\arctan ) \frac{V S}{V T}
$$

Size and density of rain streaks also normally vary with rain's intensity in an image. Therefore, we can say that with the change of these factors, the image of the rain will also change in very different ways. Hence, one of the major element that we must consider about rain is its intensity as well. With higher intensity, rain streaks will have bigger size and higher density, whereas a drizzle will have smaller streak size and lower density. Density can be generated by summing the pixel intensity (Ri) by layering rain streaks along the line of sight to the background where ' $n$ ' is the number of rain streaks and ' $i$ ' is the number of layers. By assuming the background 
$(B)$ is constant, we can model the image $(O)$ using the formula:

$$
O=B+\sum_{i}^{n}(\boldsymbol{R} \boldsymbol{i})
$$

In Figure 3.6, the dataset of rain and the clear image without rain is shown. Here, we have given 14 different images from our dataset, where rain streaks were added to the clear image shown below. As mentioned, we have processed the image by adding rain streaks to the same image several times by changing angle of rain droplets. Hence, in these 14 images in Figure 3.6, it can be seen that rain is falling at different angles in each picture with the intensity of the rain shown varying in each of the pictures.

For fog we have used image augmentation as well to create our required dataset, that we can use for training and testing purpose. As fog intensity is an important parameter for training, therefore, we will be taking random patches from all over the images, and increase lightness of the image within those patches, which will give us a nice hazy effect of fog.

Below in fig 3.5, we have shown the results. Here, 14 different images can be seen which were all created from that one clear picture provided on top of the 13 hazy ones. In each of the pictures, it can be seen that fog of different intensity is shown, some of them are hazier while some are less and some are in between. We have tried to display this varying density below in fig, which will help improve our training.

With this process completion, our dataset images are prepared according to the requirements needed for our autoencoder that we've been able feed as input and hence allowed us to move forward towards our next action, i.e., training the encoder using these processed data. 


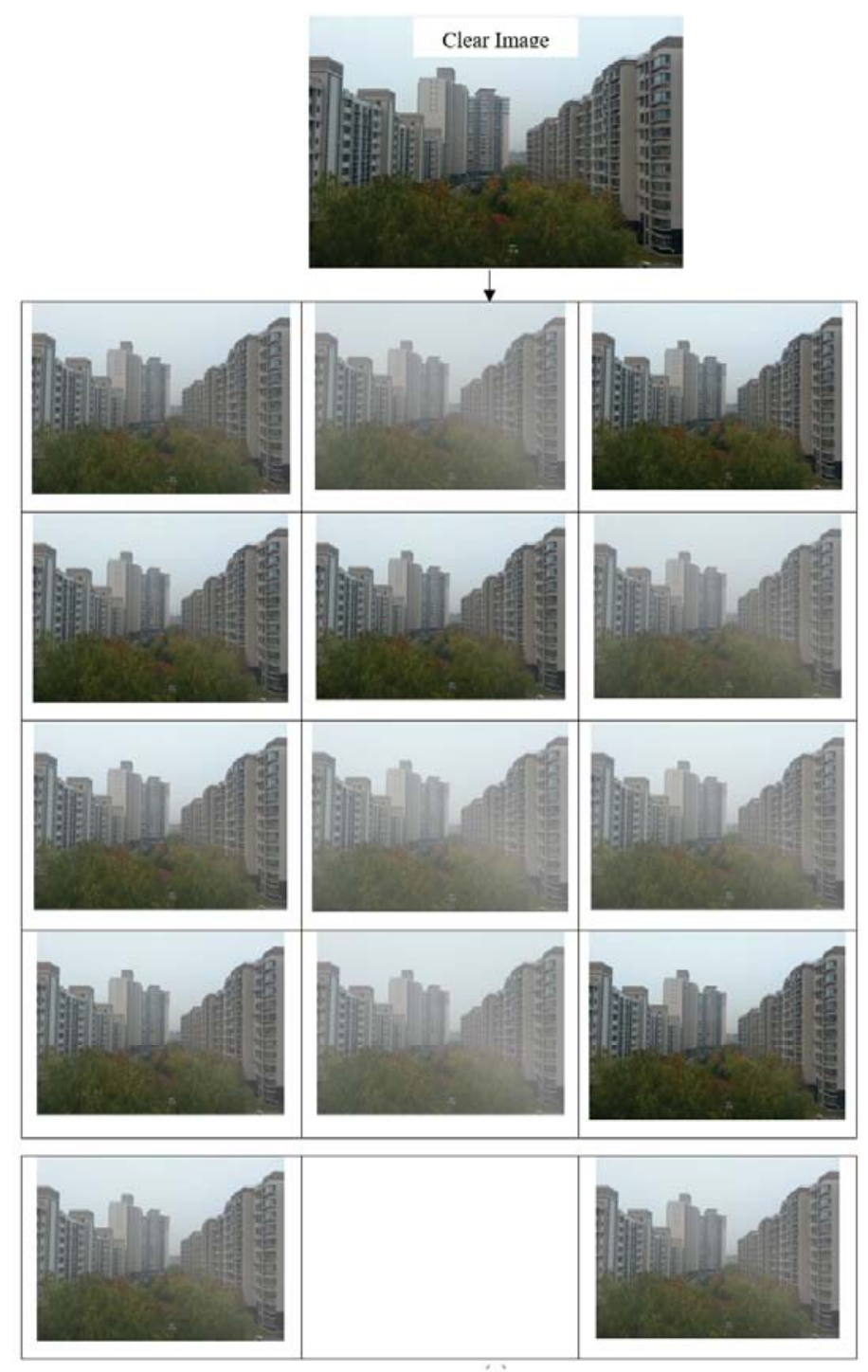

Figure 3.5: Fog Dataset (Clear image along with its 14 different density fog sample) 

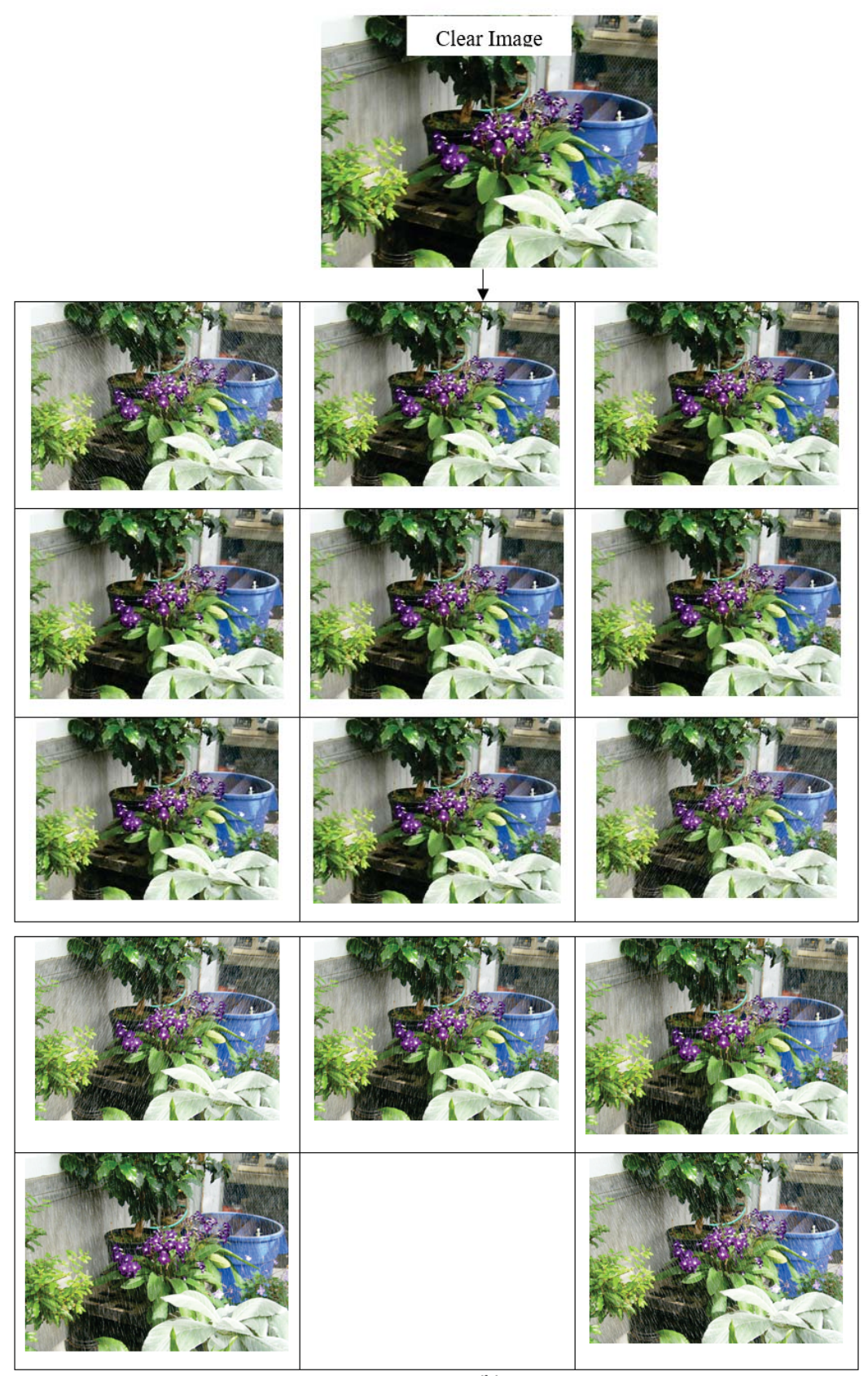

Figure 3.6: Rain Dataset (Clear image and 14 different density rain) 


\subsection{Convolutional Autoencoder}

We have opted to choose Convolutional Neural Networks for our approach, since it has yielded over the years to provide higher accuracy than any other Deep Learning model.

Convolutional Autoencoder is established on general autoencoder architecture along with the layers of convolutional encoding and decoding. The core task of this technique is to train the model enough to encode and decode a given image, by reconstructing the given input with the removal of unwanted features [24]. In addition to that, it is better suitable for image processing as the image structures are utilized to maximum capability. Generally, large chunk of information goes missing when the normal image data are chopped and stacked, but by using convolutional autoencoders, the spatial information of the input image data are preserved and the information can be extracted into Convolutional Layer.

\subsubsection{Working Principle of Autoencoder}

Autoencoding technique, implemented along with neural network, has been used to reconstruct an image which compresses the input image that has been affected by weather conditions, the middle layer (Bottleneck layer) hold the reduced representation of the input and later decompresses it to form a clear image. The output is reconstructed from this reduced representation of the input. Also, the output layer has the same dimensionality as the input layer.

\subsubsection{Autoencoder Architecture}

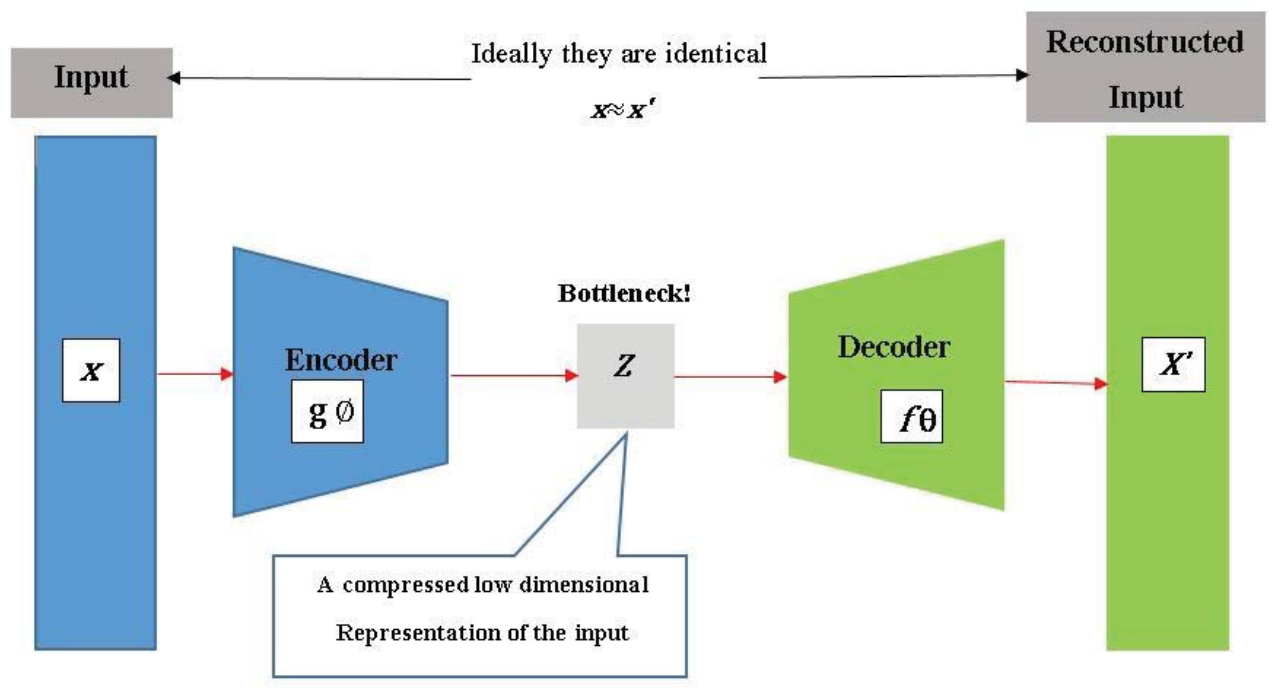

Figure 3.7: The Architecture of Autoencoder

The autoencoder comprises of three major components: Encoder, Code (Bottleneck Layer) and Decoder. As an image is fed onto the encoder, it extracts the prominent features of the image, reducing its dimensions, i.e., it encodes the input image as a 
compressed representation in a reduced dimension. The encoding layer consists of multiple layers i.e., Convolutional layers, which form this compressed version of the image which is actually the distorted version of the original image in the Code Layer. The code is a representation of the input learned through the unsupervised training procedure. The bottleneck is also called the maximum point of compression since at this point the input is compressed to its maximum. Finally, we have the Decoding layer, also called the Deconvolutional Layer, which has a similar structure to the encoder, but performs the reverse operation of the encoder. From the dimensionally compressed image, by taking the extracted pixel features, it creates an image which is seen to have removed the rain or fog effects, reconstructing each dimension of the input by passing it through the network; thus, we get a normalized image.

\subsection{Mathematical Perspective}

In Convolutional Autoencoder, the spatiality is safeguarded by distributing the weights within all the inputs. The compressed layer in the encoding part can be described by,

$$
h^{i}=s\left(x * W^{i}+b^{i}\right)
$$

where $b$ is the bias signal to be shared within the map, $W$ denotes the weight, $s$ denotes the activation function, $*$ is the $2 \mathrm{D}$ convolution and $h$ is the next layer (compressed). In the decoding portion, the reverse of the previous operation is performed to get the reconstructed image $\mathrm{x}$ by applying the similar activation function, $s$.

$$
y=s\left(h^{i} * \tilde{W}^{i}+c\right)
$$

where $c$ denotes bias per input channel and $y$ is the reconstructed version of the input image. 


\section{Chapter 4}

\section{Implementation}

\subsection{Model Training}

For our proposed model to work efficiently, where any rain or fog effects will be removed, our model needs to be trained adequately with large amount of dataset as mentioned above. The processed training image data, containing the disturbing weather factors are fed into the autoencoder along with its corresponding clear images of the dataset as label, in order to train our autoencoder.

During this training period, the autoencoder will be able to reconstruct a clear image from the affected image that we have fed as input and then compare the constructed image with the original clear image, used as label. This training will continue for a large sum of images. The more the model trains, the higher the accuracy will be with the original image. The objective of a reliable AE is to minimize the reconstructed error. 


\subsection{Building the Autoencoder and the Parame- ters Involved}

The encoder and decoder of an AE further consists of multiple layers amongst themselves as shown in Figure 4.1.

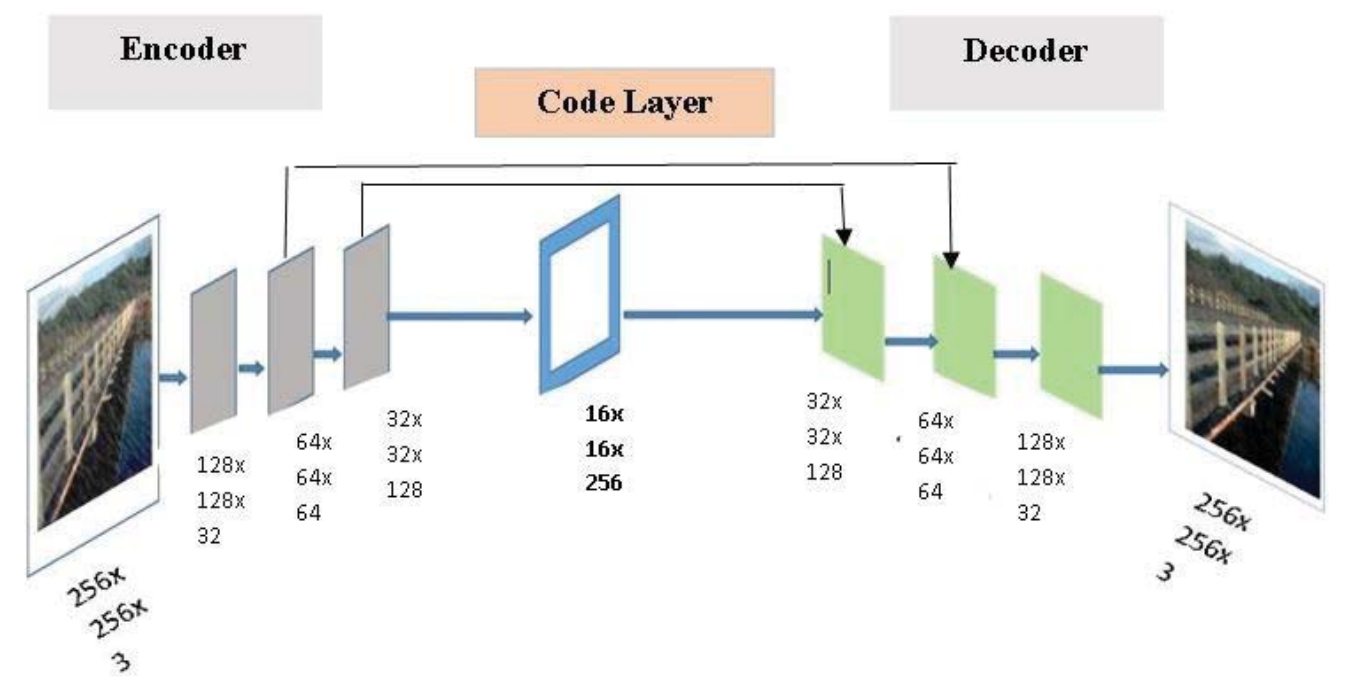

Figure 4.1: Workflow of the Model

The encoding layer is comprised of four layers where we use Convolutional layer functions along with Maxpooling. Conv2D (Convolutional function) creates tiny squares called feature maps, which preserve the pixel features and relationship within the input image. Here we have implied different number of filters of weight matrix having dimension of $3^{*} 3$ at different layers. This reduces the dimensionality of the image as the image is slightly compressed. Figure 4.1, is a short illustration of our autoencoder where we are taking input image of size $256^{*} 256$ and the 3 besides denotes the three RGB channels.

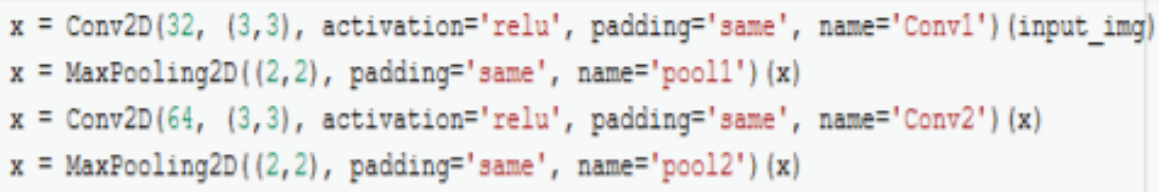

Figure 4.2: Implementation of Conv2D Maxpooling

As shown in Figure 4.2, in each layer, Conv2D is followed by Maxpooling2D. Maxpooling (MaxPooling2D) is a pooling operation which measures the maximum (largest) value in every patch of each feature map. It tends to hold the spatial presence of 
any possible pattern on the feature map of the input image i.e., output generated from Conv2D. In our model, we have used 2 by 2 window for the pool side, thus on the code, we used MaxPooling2D $((2,2))$. This procedure is to be repeated by the requirement of the hidden layers, as shown in Figure 4.3, and as it reduces the size of the image, if the target size in the Bottleneck layer is to make it smaller, $16^{*} 16$ in our model, it can be used multiple times by adjusting the parameters. The output from the first convolutional layer is then again passed to input of Conv2D along with Maxpooling2D to further compress the image until we reach the Bottleneck Layer (Code Layer), having dimension of $16^{*} 16$. To begin with, as our input image of $256^{*} 256$ pixel is fed into the autoencoder, the first layer compresses it into $128^{*} 128^{*} 32$, since the filter used is 32 . As shown in Figure 4.1, it is fed into another layer having filter of 64 and now the feature extraction results an output of $64 * 64^{*} 64$. By repeating this procedure for next two layers, we reach the Bottleneck layer (Code layer) where the dimensionality is $16^{*} 16^{*} 512$.

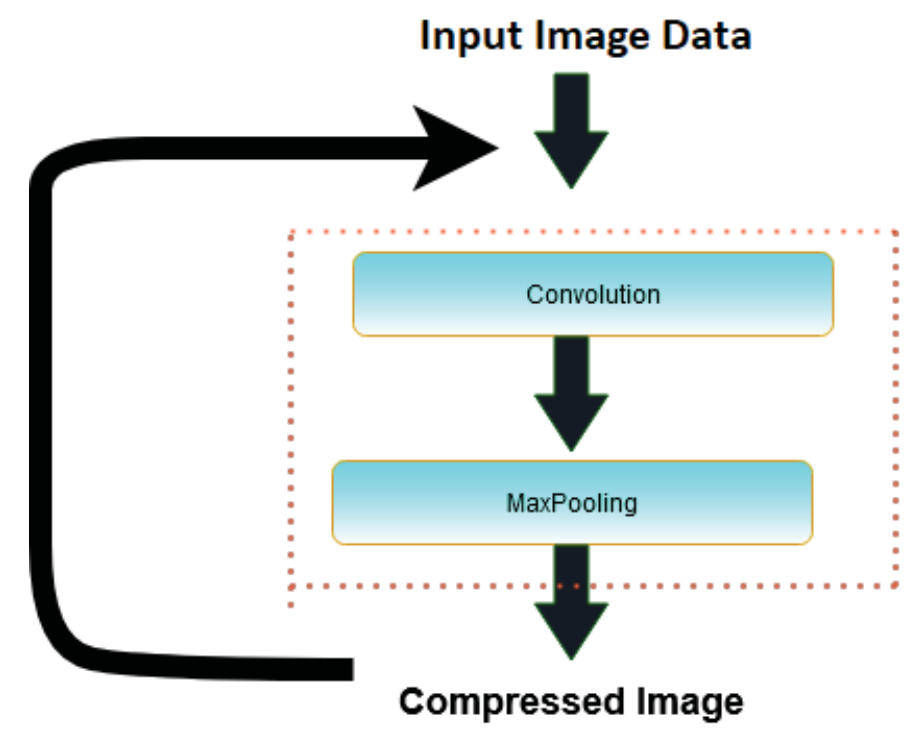

Figure 4.3: Addition of Multiple Conv2D and Maxpooling

\section{Code Layer}

Figure 4.1 shows, this layer holds the compressed representation of our data, as it drives the network to create a compressed form of the input image. From the input image of $256^{*} 256^{*} 3$, the dimensionality has been reduced to $16^{*} 16^{*} 512$. This holds the spatial features extracted from the input and will now be used by the Decoder Layer to reconstruct the image.

\section{Decoder}

At decoding part, the model is to take the compressed data from the Code layer image as input and perform the exact mirror operations to the encoding part, illustrated at the decoder of Figure 4.1. Similar to encoder part, decoder comprises of four layers where in each layer we are enlarging the dimension of the image. In each layer, we are using Conv2DTranspose and UpSampling. From the bottleneck layer, the $16^{*} 16$ dimensional data is taken and the output of this layer is $32^{*} 32$. Likewise, the generated output is passed onto another layer to generate $128^{*} 128$ image and finally by passing through the last layer of the decoding layer we get our 
desired reconstructed image. Here, we had to implement the parameters (strides and a number of filters) in such a way that the reconstructed image is of the same dimensionality as of the input image and this was done by ensuring that the number of hidden layers in the encoder is the same as the number of layers in the decoder. Lastly, we used Conv2D with a depth of three as we want to produce RGB images.

\section{Formation of Feature Maps}

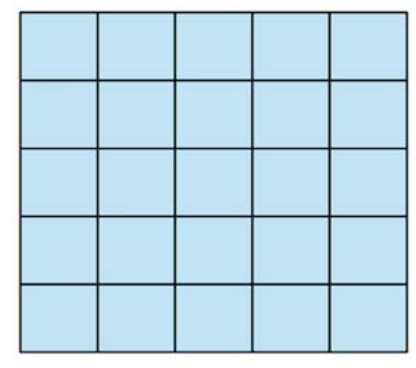

Input

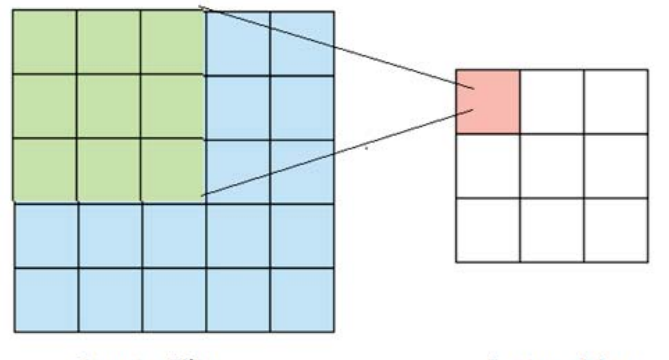

Input x Filter
Feature Map

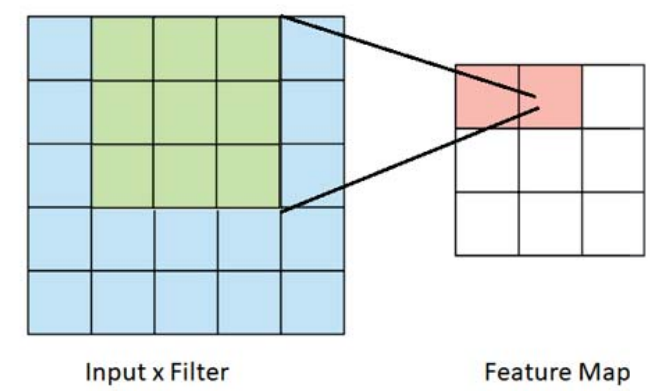

Feature Map

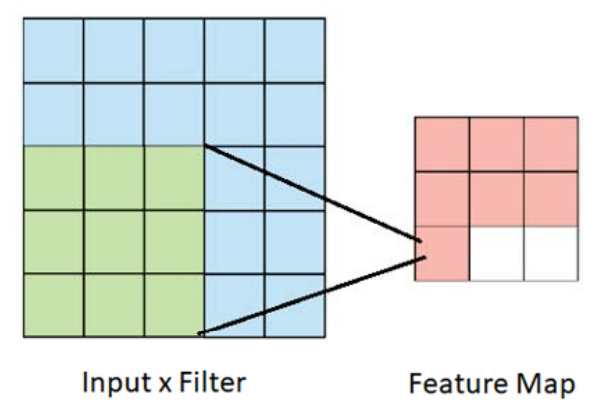

Figure 4.4: Basic Formation of Feature Map

Figure 4.4 illustrates how the feature map is formed in a simpler form by the application of convolutional operation on $2 \mathrm{D}$ input. The filter is passed over the image as shown in the figure. For each location, matrix multiplication is performed and its sum forms part of the feature map. As we can see, having stride of 1 , the filter is passed over different region of the input image, and this forms the feature map, containing the information of the pixel in a compressed form. However, since we are using RGB images as input, it has three channel and hence there are improvisation to the procedure. For the three channels, the filter to be used much also have three 
channels. Also, as mentioned earlier, since we are using multiple number of filters, for each filter a feature map is formed and the results are stacked together to form a 3-dimensional feature map as shown in Figure 4.5 and 4.6, where the different colors indicate usage of multiple filters.

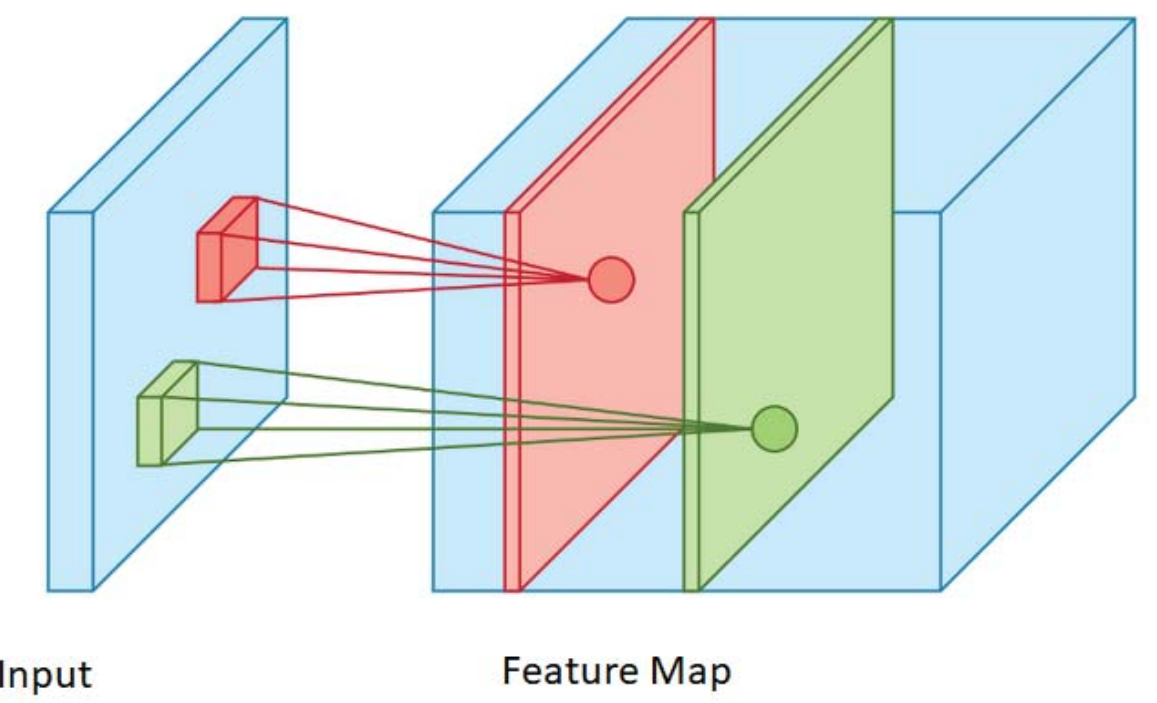

Figure 4.5: Detailed Formation of Feature Map for Three Channel (RGB) Image
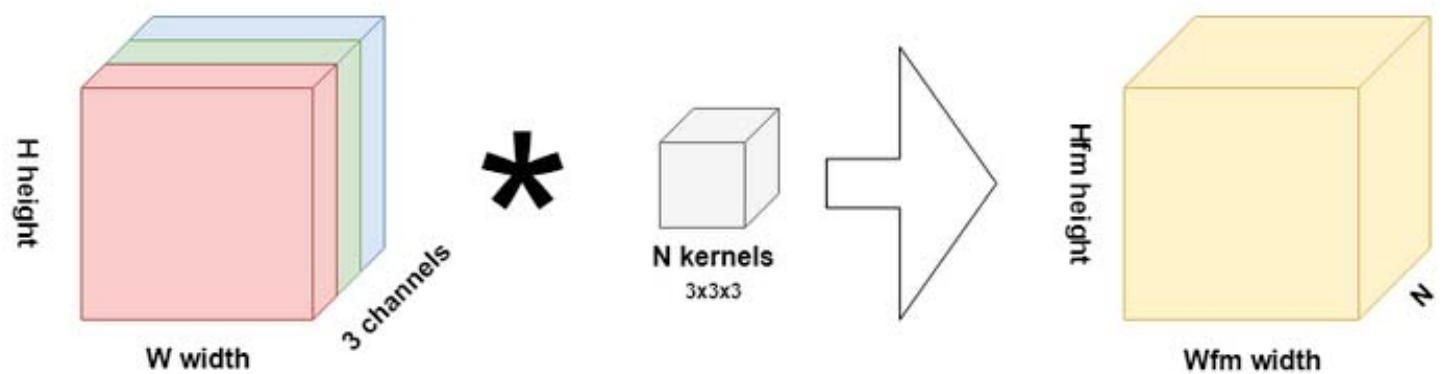

Figure 4.6: Overview of the Feature Map 


\section{Parameters}

\section{Padding}

Padding is amongst one of the noteworthy parameters involved to train our model, taken by Conv2D function. As shown in Figure 4.7, as the kernel of stride 1 moves over the input image, the corner pixel information is not extracted as well as compared to the middle pixels, which will result in lack of information on the feature map. To overcome such problem, we need padding. In our model, we have used padding $=$ same. Padding works by the extension of the area of the input image pixels while processing it. Small extensions are done on the frame of the image for the kernel to cover the image, preventing the loss of spatial information on the feature map. In most cases, padding increases the scope of accurate evaluation of image, by preventing shrinkage of the image and also this ensures that pixels at the border of the image have similar contribution to the feature map compared to the pixels in the middle of the image. Though there are different types of padding but we opted to keep the padding as same due to large training dataset, since the training time will be affected. Furthermore, padding of other type would be helpful if our image was of uneven pixel size in terms of width and height; but our images are of $256^{*} 256$ pixels.
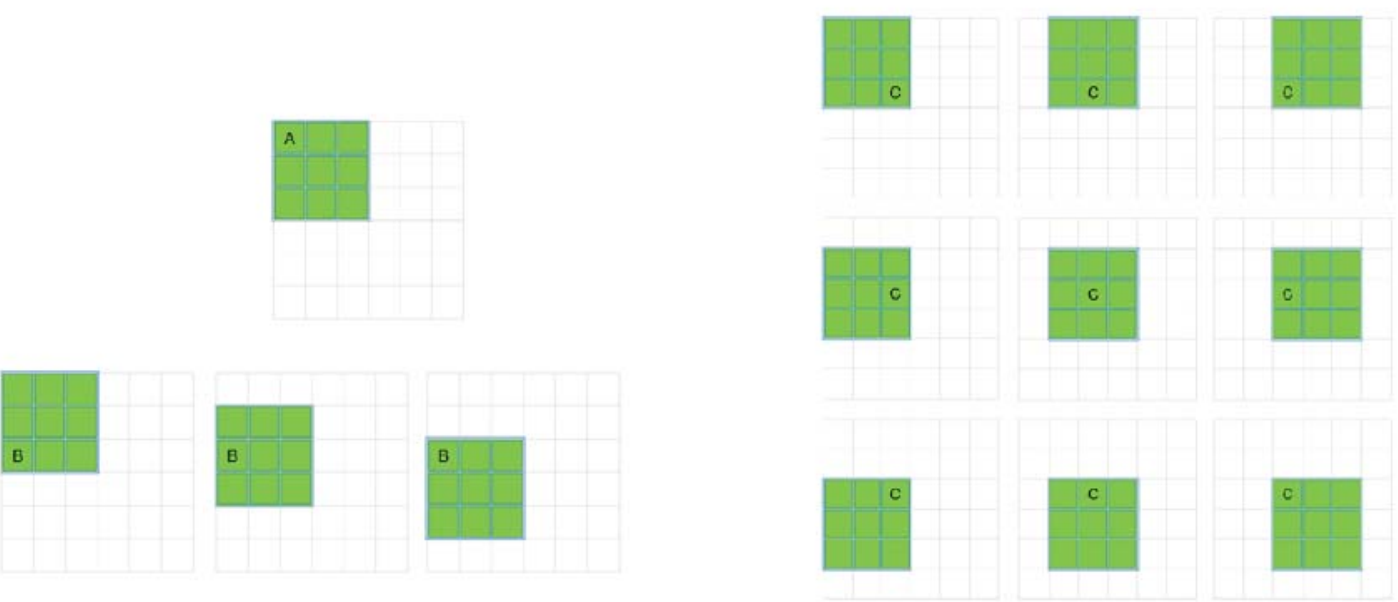

Figure 4.7: Simulation of Filters Over Image Without Padding 

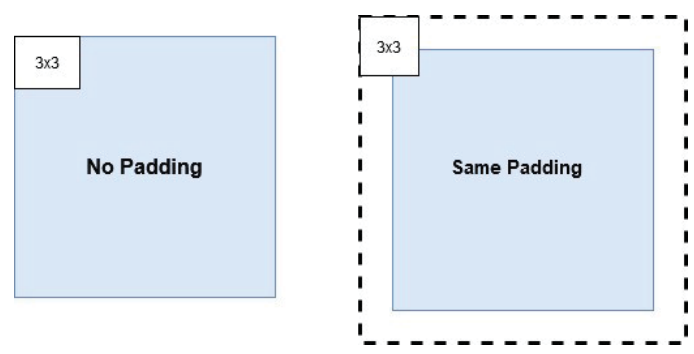

Figure 4.8: Consequences of Adding Padding

\section{Stride}

Stride is a process for the compression of pictures in neural networks. Stride is a measure of movement of kernel over the picture. Normally, as the stride is moving, the subsequent yield will be more modest. Stride is a boundary that works related to padding, the element that adds clear, or void pixels to the casing of the picture to take a limited decrease of size in the yield layer into consideration. It determines the number of block that the filter will shift. Generally, it is a method of expanding the size of a picture, to check the way that stride decreases the size. Padding and stride are the central boundaries of any convolutional neural organization.

\section{Activation Function}

ReLU (Rectifier Linear Unit) function has been used in our model. ReLU is a piecewise linear function. This function will give output only if the input is positive otherwise it will show zero. The benefits of ReLU are sparsity and reduction in chance of vanishing gradient. Besides, ReLU is among one of the quickest, as it doesn't actuate all neurons simultaneously. ReLU activation function (ReLU) is defined as:

$R(x)=\max (0, x)$

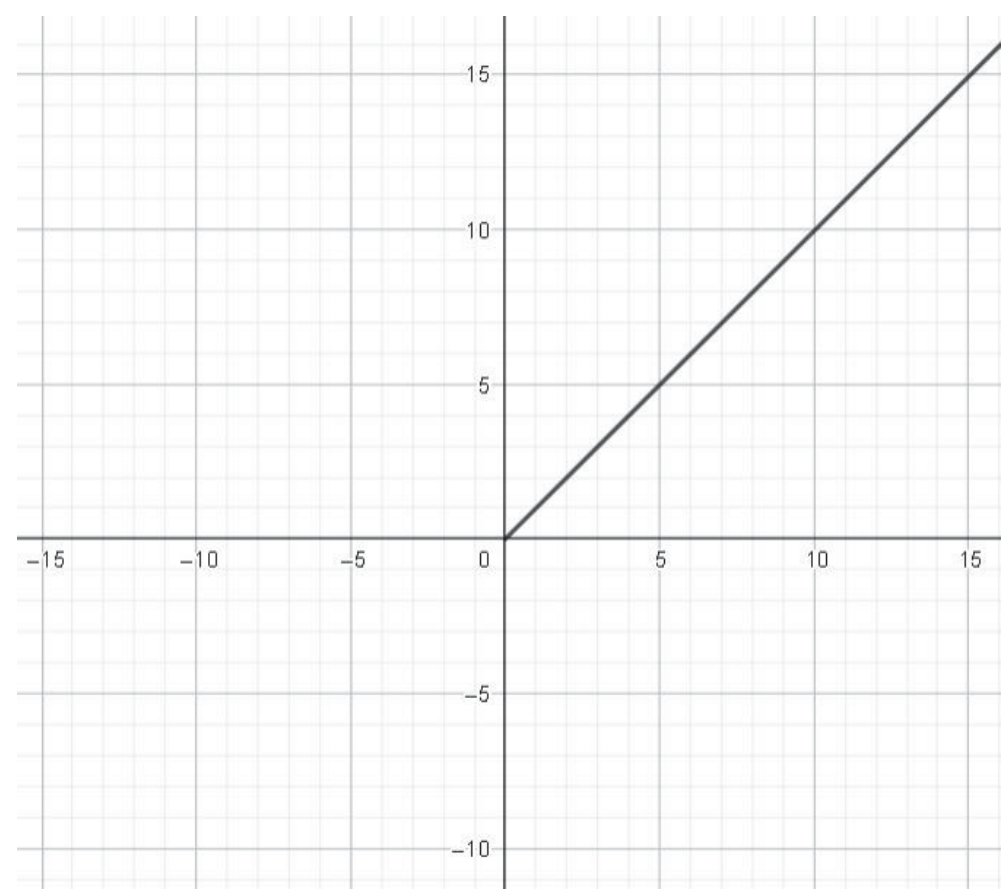

Figure 4.9: Graphical Representation of ReLU 
One reason that this function is added into a neural network is to assist the network in learning complex patterns in the data. The figure shows that the output value can either be zero or any positive value, which can be expressed by the equation. This function acquaints nonlinear real-world properties with neural network. Activation function like ReLU have two main part - helping a model account for interaction effects and non-linear effects. The reason we are using ReLU activation function over other function as it has no complex math and its computation time is less than other activation function. For that ReLU will take less time to train our model. Besides that, we used ReLU for its sparsity as it gives zero value for all the negative value and has no range of infinity of positive value, as shown in Figure. Moreover, whenever the matrix multiplication occurs, ReLU ensures that the feature map does not contain any negative value. Moreover, it solves the vanishing gradient problem. The ReLU work has a derivative of 0 over a large portion of its range. For positive value, the derivative is 1 . When preparing on a sensible sized batch, there will typically be some information that directs giving positive qualities toward some random hub. So, the normal subsidiary is seldom near 0, which permits gradient descent to continue advancing. In addition to that, ReLU is better than Sigmoid or tanh, as it is faster but most importantly it has better accuracy.

\section{Optimization Function}

Adam Optimizer is an optimization algorithm utilized while compiling the autoencoder. We have used Adam Optimizer, which computes adaptive learning rate for each parameter. Adam optimization algorithm is an expansion to stochastic gradient descent in light of versatile assessment of first order and second order moments for training deep learning model. Adam consolidates the AdaGrad and RMSProp calculations to give an enhancement calculation to manage sparse gradients on noise issue. Adam Optimizer is a versatile learning rate technique, which implies, it figures singular learning rates for various parameters. In our Keras api we used Adam optimizer to get most accurate value from our model. Beside that it take less memory, have straight forward implementation and have efficient output. As we have a large dataset the Adam optimizer is the best solution for us. It is appropriate for rainy/hazy noise in image and its hyper parameters have instinctive understanding and usually require small tuning. The progression size taken by the Adam in every cycle is roughly limited the progression size hyper parameter. Step size of Adam update rule is invariant to the size of the slope, which assists a great deal while experiencing territories with little gradients. It is similar and an extension to Adadelta plus momentum (which smooths gradients based on accumulated first-order information); having a learning rate of 0.001 . 


\section{Loss Function}

In our model, the encoding and decoding involve some loss of information in the process and to keep a track of it we have used Mean Squared Error (MSE). Firstly, the difference between the reconstructed output image and original version of the input image is calculated, and the MSE is calculated by averaging the square of the calculated error [24]. MSE can be calculated by the formula,

$$
\boldsymbol{M S E}=\frac{1}{W * H} \sum_{i=0, j=0}^{W-1, H-1}[R(i, j)-G(i, j)]^{2}
$$

where $R(i, j)$ and $G(i, j)$ denote reconstructed image and the original image respectively, $\mathrm{W}$ and $\mathrm{H}$ are the width and height of these images. The row and column of the original and reconstructed image are denoted by $\mathrm{i}$ and $\mathrm{j}$. MSE embraces the variance along with the bias of the estimate [15]. Compared to other loss function, MSE is suitable as it simply compares between two images and if the two images are exactly same then the difference would be zero, indicating that there is no difference between the images [4]. Throughout the process, our goal was to minimize this value. 


\section{Chapter 5}

\section{Result and Analysis}
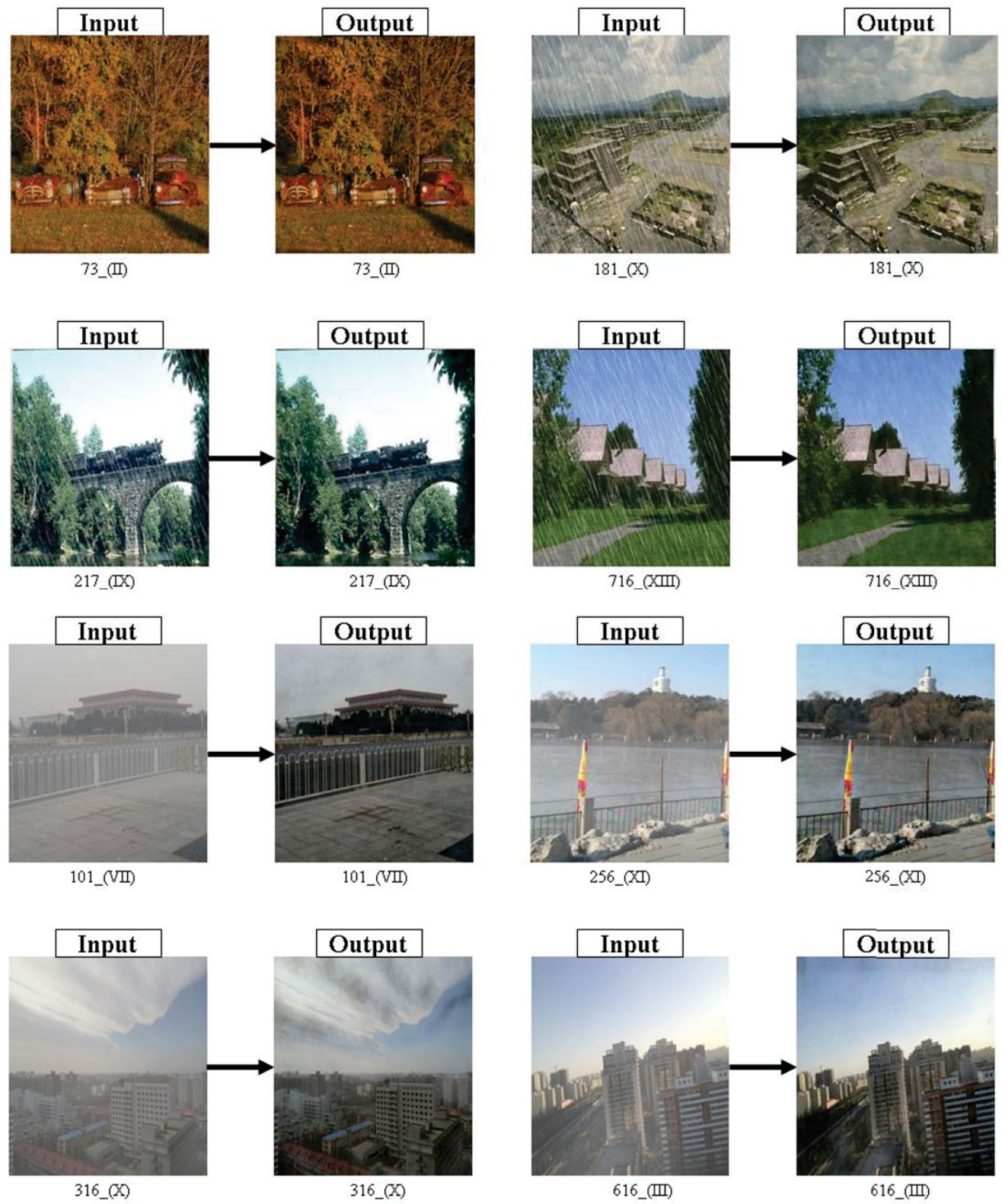

Figure 5.1: Illustration of Random Test Images along with Corresponding Output 
In our thesis we have taken fourteen (xiv) different orientation images to train our model. Then we fed that data into our autoencoder's model. As we can demonstrate that with a small pictorial illustration as in Figure 5.2.

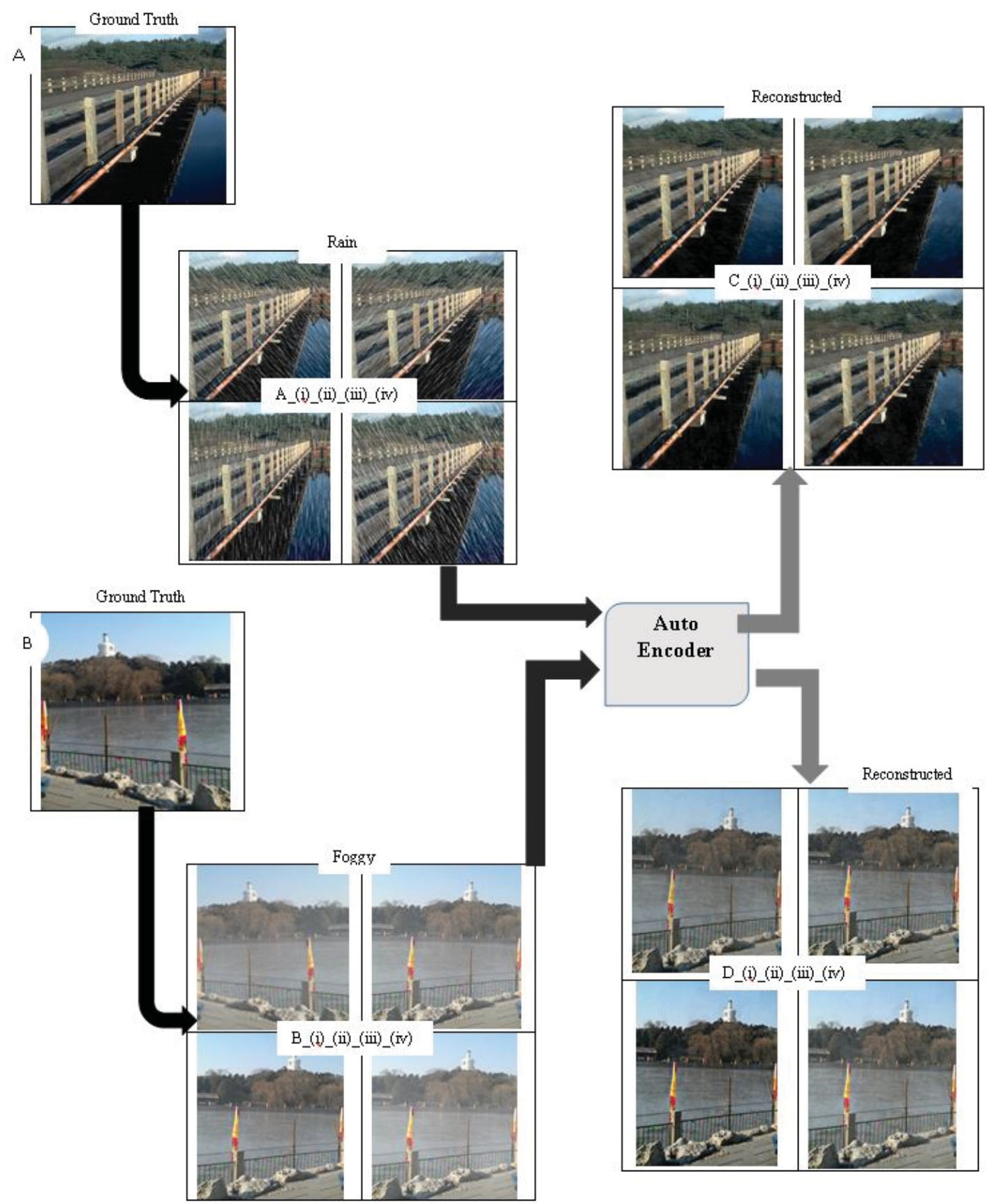

Figure 5.2: Reconstruction of Image Using Autoencoder

For keeping this pictorial demonstrate small and simple we are showing only four (iv) images has been fed into the autoencoder model but in our actual code we have added fourteen images. In the figure 5.2, we can see ground truth A has four different orientation raining scenarios. They are A(i), A(ii), A(iii) and A(iv). These four images are then fed into the autoencoder for model training. Upon training the autoencoder gives us the result C(i), C(ii), C(iii) and C(iv). These reconstructed images are actually the clear image result of the rainy image. Similarly, we can 
see ground truth B has four different density fog scenarios. They are B(i), B(ii), $\mathrm{B}$ (iii) and $\mathrm{B}(\mathrm{iv})$. These four images are then fed into the autoencoder for model training. Upon training the autoencoder gives us the result $\mathrm{D}(\mathrm{i}), \mathrm{D}(\mathrm{ii}), \mathrm{D}$ (iii) and $\mathrm{D}(\mathrm{iv})$. These reconstructed images are actually the clear image result of the foggy image. So, this is how our actual autoencoder training and reconstruction of the image occurring in the actual code.

\section{Structural Similarity Index}

Structural Similarity Index, known by SSIM, is used to check the reliability of our model. It is salient for us to evaluate and analyze our method of data training. SSIM looks at a group of pixels which makes it more effective. It evaluates images considering sensitive changes in local structure. Using SSIM our goal is for the network to learn to produce distortion free and structurally correct images. The SSIM index is calculated between two windows $x$ and $y$ of common size $\mathrm{W}^{*} \mathrm{~W}$ is:

$$
\operatorname{SSIM}(\mathbf{x}, \mathbf{y})=\frac{\left(2 \mu_{x} \mu_{y}+c_{1}\right)\left(2 \sigma_{x y}+c_{2}\right)}{\left(\mu_{x}^{2}+\mu_{y}^{2}+c_{1}\right)\left(\sigma_{x}^{2}+\sigma_{y}^{2}+c_{2}\right)}
$$

Here, $x, y$ are the $\mathrm{W}^{*} \mathrm{~W}$ frame taken from ground truth and reconstructed image. is the average of $x$, is the average of $y$, is the variance of $x$, is the variance of $y$, is the covariance of $x$ and $y$, and are two variables to stabilize the division with weak denominator and is the dynamic range of the pixel values.

SSIM examines the identicality with the original image [14], here in our model, it will be ground truth of the image. Calculated value of SSIM varies from 0 to 1 , where the closer the value is to 1 , the better is the output generation with respect to the original ground truth image. SSIM is helpful in assessing the quality of the reconstructed image [4]. 


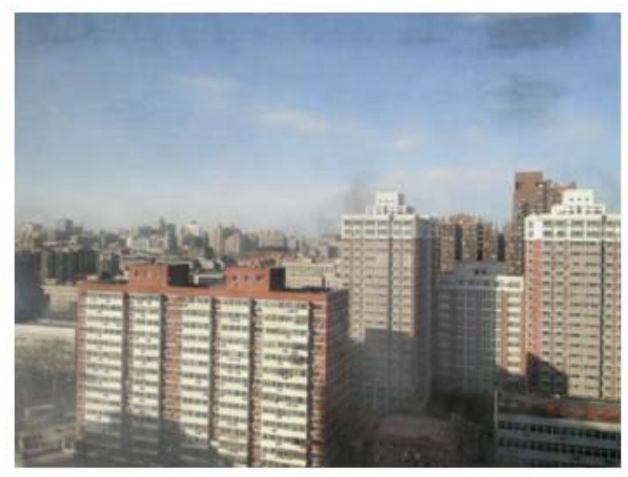

(a)

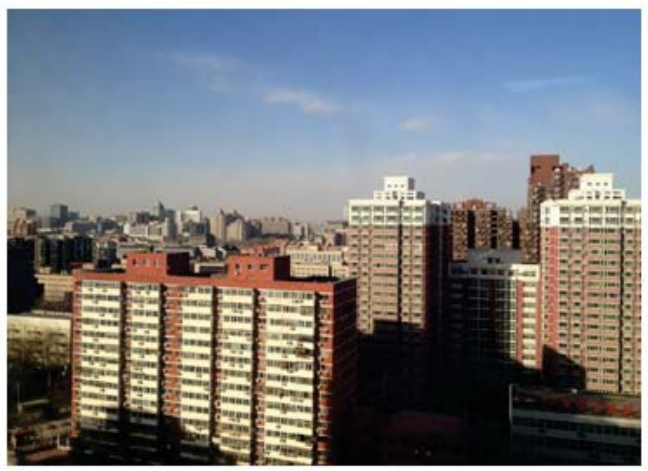

(b)

Figure 5.3: Comparison of Output and It's Ground Truth (Original Image): (a) Reconstructed Image (b) Ground truth

The visual comparison between (a) the reconstructed image by the model and (b) the actual image (ground truth) is shown in Figure 5.3. The calculated SSIM value is 0.90 which depicts the accuracy of our model with the testing dataset. 


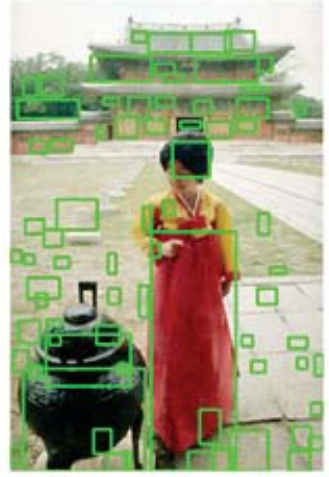

(a)

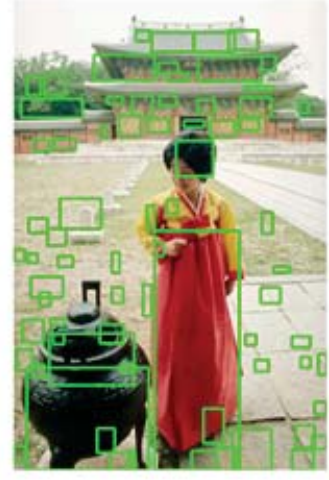

(b)

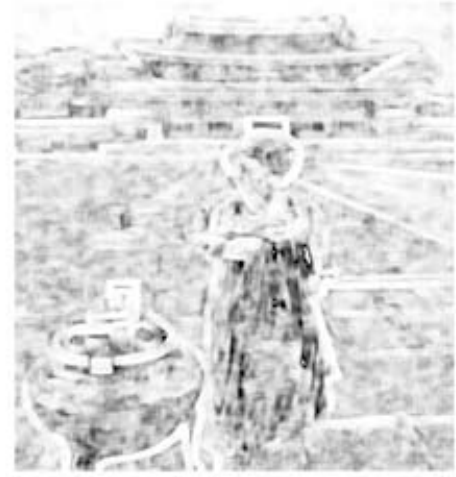

(c)

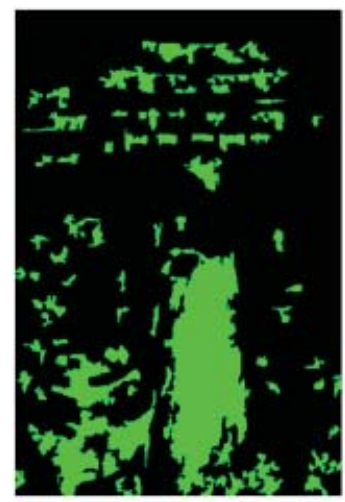

(d)

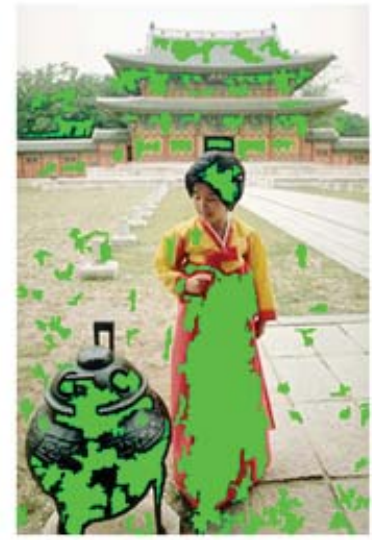

(e)

Figure 5.4: Detailed Analysis of Rainy Images (a) reconstructed image, (b) ground truth, (c) differences plot in grayscale, (d) mask plot and (e) filled mask on clear image

In both the images, Figure 5.4 (a) and (b) for raining condition and Figure 5.5 (a) and (b) for fog condition, the green boxes are seemed to be in identical position, this denotes that our algorithm for SSIM is finding the dissimilarities within these regions. For better visualizing, grey plotting is done, as shown in Figure 5.4 (c) and Figure 5.5 (c), where places of similarity are whitened and on dissimilarity regions it forms grey. Furthermore, we have used masking as shown in 5.4 (d) and 5.5 (d) where the green plots that can be seen are the dissimilarities. The dissimilarities are then plotted in the main image, to see the places of having certain mismatches. 


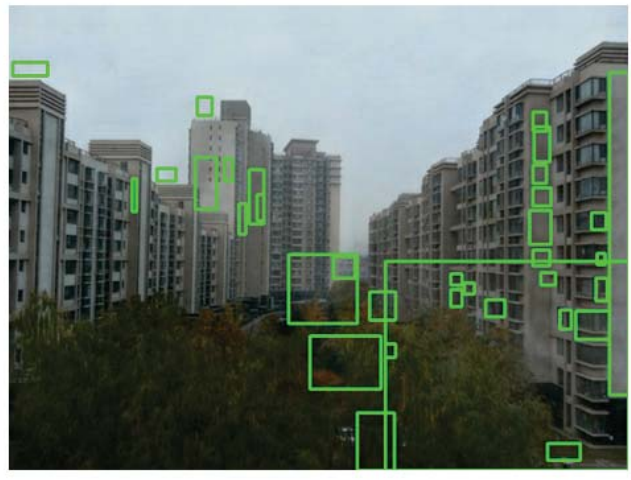

(a)

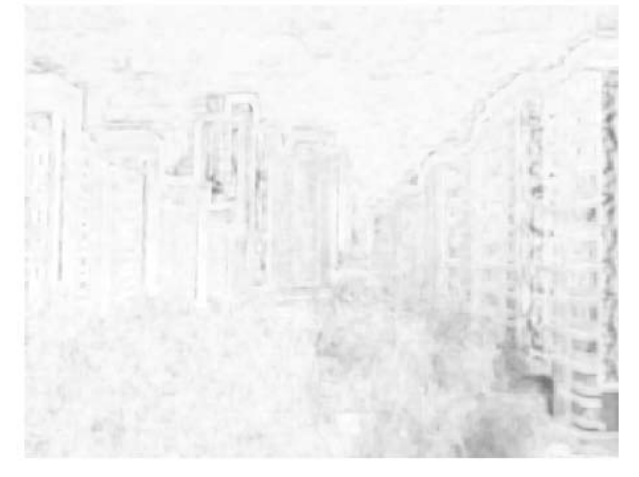

(c)

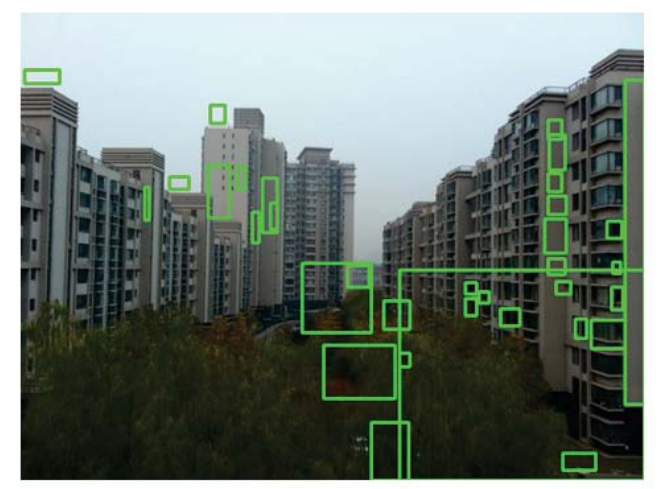

(b)

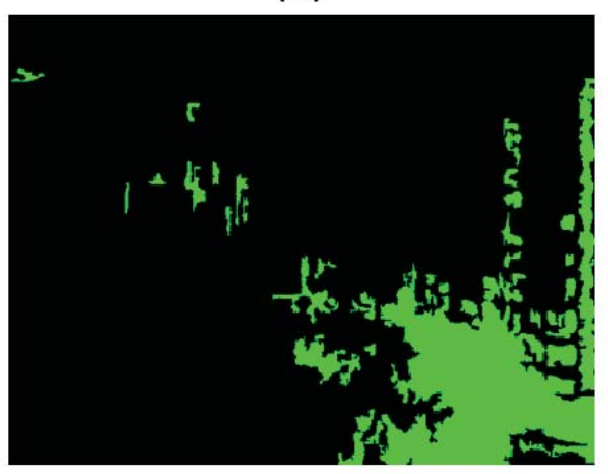

(d)

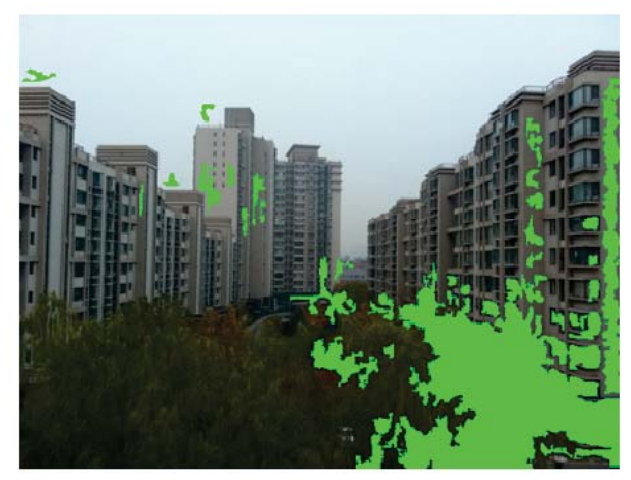

(e)

Figure 5.5: Detailed Analysis of Foggy Image (a) reconstructed image, (b) ground truth, (c) differences plot in grayscale, (d) mask plot and (e) filled mask on clear image 


\section{PSNR (Peak signal to noise ratio)}

To assess the quality of the output image of our model, we have used Peak signal to noise ratio methodology. It is the ratio of maximum signal's energy and the noise of the newly formed image, where the signal is data of actual image which is stated as the ground truth and the noise denotes the error in the image. It is amongst one of the prominent methods for evaluating the quality of reconstructed image [15], and it is expressed in decibel. The higher the value the better will be the quality of the image.

$$
\boldsymbol{P S N R}=10 * \log _{10} \frac{M A X_{I}^{2}}{M S E}
$$

where, $M A X i$ is usually 255 , which is maximum pixel value and $M S E$ is Mean Squared Error. Figure 5.6 and 5.7 show the PSNR value comparison of both rainy and foggy image. It shows the original image (which is the ground truth), the rainy or foggy image and the reconstructed output. 

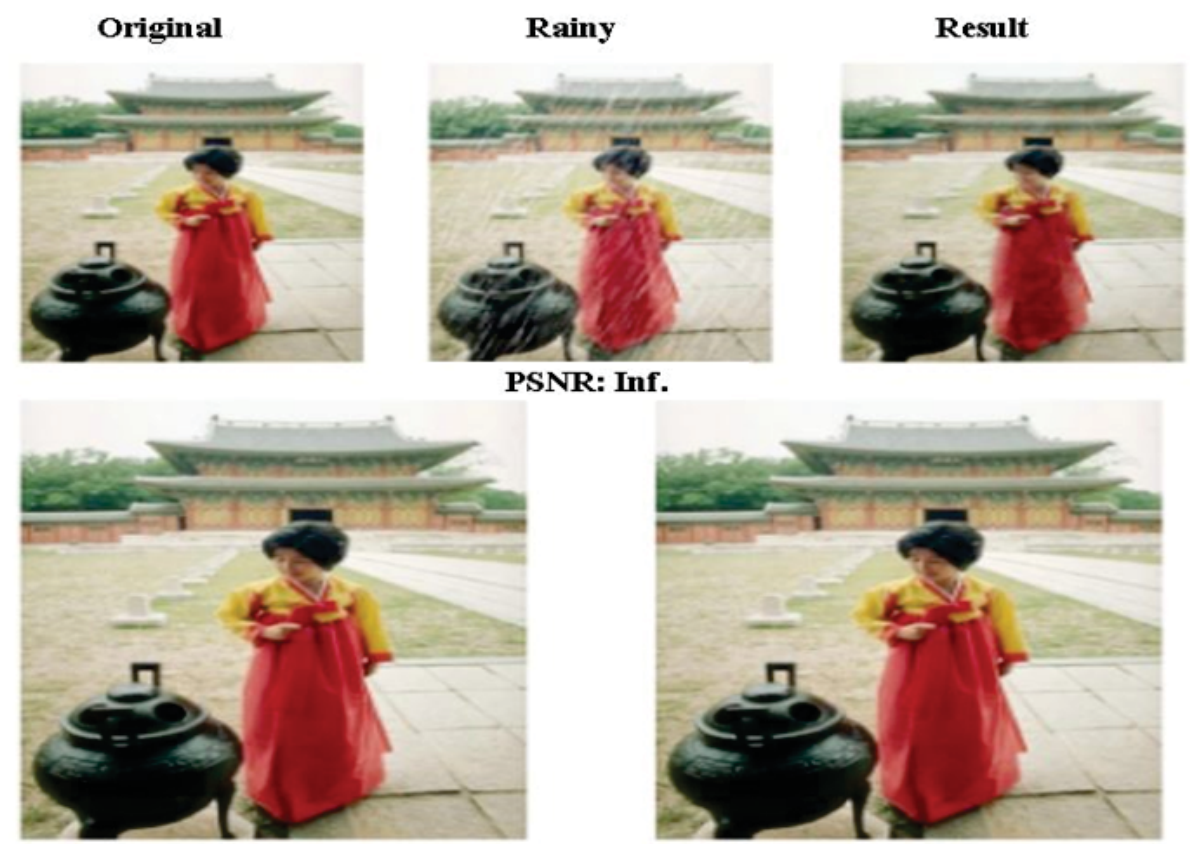

PSNR: Inf.

PSNR: 31.12
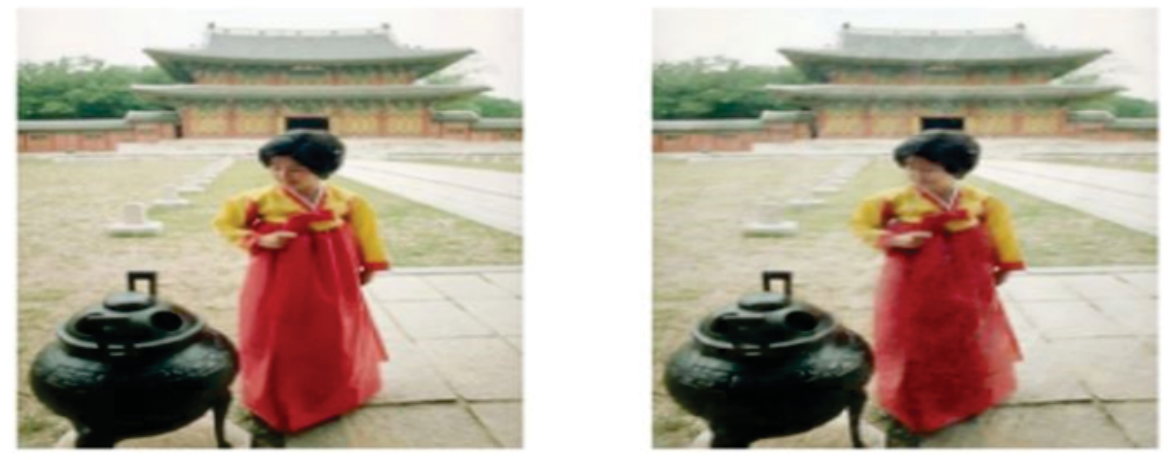

Figure 5.6: PSNR of Rainy Image 

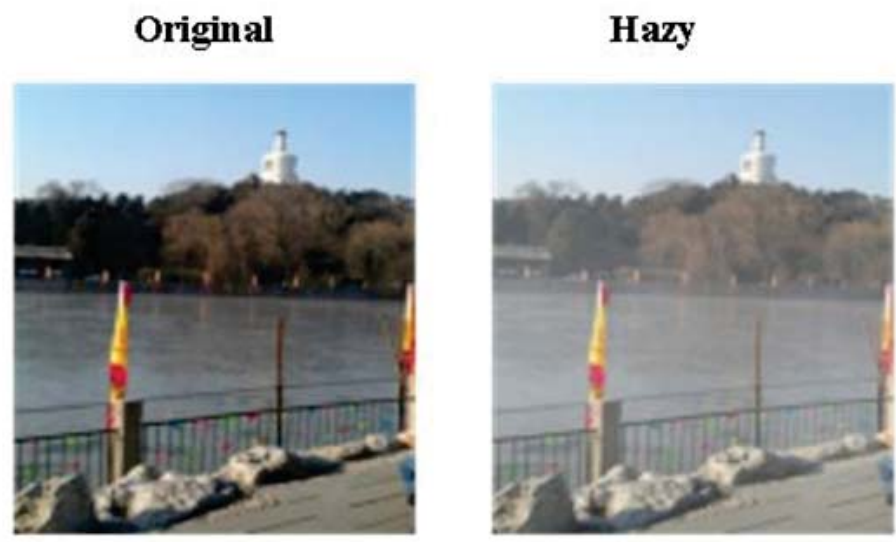

Result

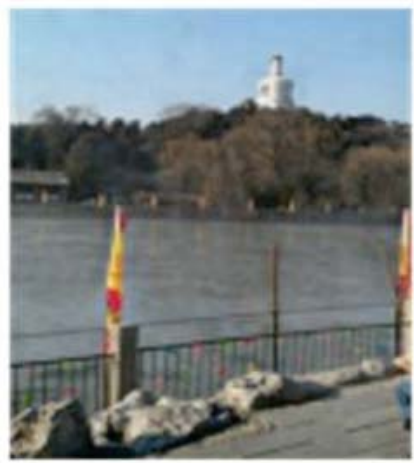

PSNR: Inf.
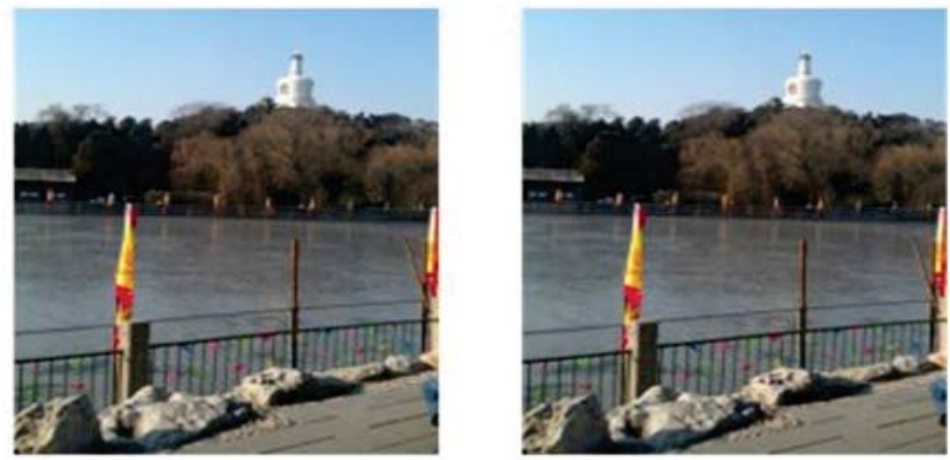

PSNR: 28.85
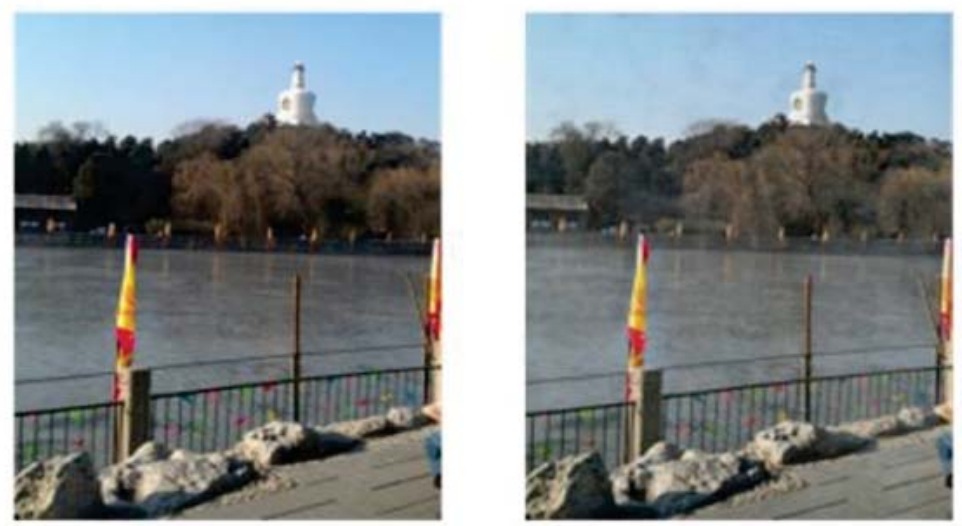

Figure 5.7: PSNR of Foggy Image 


\section{Chapter 6}

\section{Conclusion}

The past 100 years have been the most dramatic technological upheavals to life than in all of human history. And it is believed, the next 100 years is set to pave the way for a multi-generational leap forward. This will be at the hands of artificial intelligence. Since everything coming out of the technology world today seems to have some element of artificial intelligence or machine learning to it. We are therefore moving rapidly into an era of AI; from voice-powered personal assistants like Siri and Alexa, to more underlying and fundamental technologies such as behavioral algorithms, suggestive searches and autonomously-powered self-driving vehicles boasting powerful predictive capabilities. Such code-driven systems have spread to more than half of the world's inhabitants in ambient information and connectivity, offering previously unimaginable opportunities. In this uprise of Artificial Intelligence, computer vision plays a vital role in order to interconnect material world with the technological. So, keeping that in our mind we have come up with a proposed idea to make computer vision not only effective, but also more accurate and more intelligent. Our paper "A Color Vision Approach Considering Weather Conditions Based on Autoencoder Techniques Using Deep Neural Networks" tried to utilize this opportunity and work on a major hindrance in the way of a successful image analyzation, that is, we have come up with a solution to remove weather condition from images which will allow many smart technologies such as self-driving autonomous vehicle and infrastructure monitoring for security to operate in more adverse weather condition while eliminating chances of catastrophic events caused by the slightest errors. Hence, the purpose of our thesis paper is to develop a model which considers different types of environmental elements like rain and fog concurrently to enable composite analyzation and normalization of images. To serve this motive, we have used an autoencoder technique that consists of three processes of encoding, code and decoding, where a rainy or foggy image that we have processed using image augmentation and resizing algorithms is fed to the encoding layer and by reducing its dimensions, the target features of the image is extracted. In the code layer (bottleneck layer), the compressed version of the image is formed and it conveys further depth to our final output. And lastly, the Decoding layer takes the extracted pixel features from dimensional compressed image and creates an image that has the rain and fog effects removed. As we are aiming for accuracy, we chose Convolutional Neural Networks for our approach, since it provides higher accuracy than any other Deep Learning model. Humans have always fixated themselves on improving life across every spectrum, and basically, the use of our technology aims 
to become the vehicle for doing just that.

\subsection{Limitations}

COVID-19 has been a massive hindrance in conducting our research as we could not have face to face meetings and had to rely on online tools. It also denied us the use of well-equipped computer laboratories of our university and since we do not own any high-end computers, it was difficult to polish off our results. If we could have access to higher-end computers we would have been able to generate precise results a lot faster which would enable us to train the model even more for exemplary results.

\subsection{Future Work}

Despite having very promising results, our model can still be improved with further enhancements for better, easier and more approachable usability. Some potential enhancements are as follows:

- The model can be integrated with not only weather factors but also other environmental elements like intensity of light for operating in variable lighting conditions or it could also be integrated with night-vision accessories for operating at night.

- For security applications the model can be used to detect robbery or traffic rule violations and can also be integrated with alarm systems to trigger or inform the police and store the correlating information of the incident.

- It can also be implemented in factories or stores for monitoring which would ensure safer environment for employees or customers or can also be set to monitor manufacturing defects or to reduce quality control mistakes. 


\section{Bibliography}

[1] J. D. Crisman and C. E. Thorpe, "Unscarf-a color vision system for the detection of unstructured roads.," in ICRA, 1991, pp. 2496-2501.

[2] J. Zheng, K. P. Valavanis, and J. M. Gauch, "Noise removal from color images," Journal of Intelligent and Robotic Systems, vol. 7, no. 3, pp. 257-285, 1993.

[3] S. G. Narasimhan and S. K. Nayar, "Chromatic framework for vision in bad weather," in Proceedings IEEE Conference on Computer Vision and Pattern Recognition. CVPR 2000 (Cat. No. PR00662), IEEE, vol. 1, 2000, pp. 598605.

[4] A. Z Wang, H. C Bovik, R. Sheikh, and E. P. Simoncelli, "Image quality assessment: From error visibility to structural similarity," IEEE transactions on image processing, vol. 13, no. 4, pp. 600-612, 2004.

[5] P. Baldi, "Autoencoders, unsupervised learning, and deep architectures," in Proceedings of ICML workshop on unsupervised and transfer learning, 2012, pp. 37-49.

[6] J. Xie, L. Xu, and E. Chen, "Image denoising and inpainting with deep neural networks," Advances in neural information processing systems, vol. 25, pp. 341-349, 2012.

[7] L. Gondara, "Medical image denoising using convolutional denoising autoencoders," in 2016 IEEE 16th International Conference on Data Mining Workshops (ICDMW), IEEE, 2016, pp. 241-246.

[8] M. Koziarski and B. Cyganek, "Image recognition with deep neural networks in presence of noise-dealing with and taking advantage of distortions," Integrated Computer-Aided Engineering, vol. 24, no. 4, pp. 337-349, 2017.

[9] X. Li, T. Pang, B. Xiong, W. Liu, P. Liang, and T. Wang, "Convolutional neural networks based transfer learning for diabetic retinopathy fundus image classification," in 2017 10th International Congress on Image and Signal Processing, BioMedical Engineering and Informatics (CISP-BMEI), IEEE, 2017, pp. $1-11$.

[10] G. Litjens, T. Kooi, B. E. Bejnordi, A. A. A. Setio, F. Ciompi, M. Ghafoorian, J. A. Van Der Laak, B. Van Ginneken, and C. I. Sánchez, "A survey on deep learning in medical image analysis," Medical image analysis, vol. 42, pp. 6088, 2017.

[11] K. G. Lore, A. Akintayo, and S. Sarkar, "Llnet: A deep autoencoder approach to natural low-light image enhancement," Pattern Recognition, vol. 61, pp. 650-662, 2017. 
[12] S. S. Roy, M. Ahmed, and M. Akhand, "Classification of massive noisy image using auto-encoders and convolutional neural network," in 2017 8th International Conference on Information Technology (ICIT), IEEE, 2017, pp. 971979.

[13] S. Arjomand Bigdeli and M. Zwicker, "Image restoration using autoencoding priors," in Proceedings of the 13th International Joint Conference on Computer Vision, Imaging and Computer Graphics Theory and Applications, SCITEPRESS, vol. 5, 2018, pp. 33-44.

[14] D. Asamoah, E. Ofori, S. Opoku, and J. Danso, "Measuring the performance of image contrast enhancement technique," International Journal of Computer Applications, vol. 181, no. 22, pp. 6-13, 2018. DOI: 10.5120/ijca2018917899.

[15] D. Asamoah, E. O. Oppong, S. O. Oppong, and J. Danso, "Measuring the performance of image contrast enhancement technique," International Journal of Computer Applications, vol. 181, no. 22, pp. 6-13, Oct. 2018, ISSN: 0975-8887. DOI: 10.5120/ijca2018917899. [Online]. Available: http://www.ijcaonline.org/ archives/volume181/number22/30015-2018917899.

[16] C. Ferles, Y. Papanikolaou, and K. J. Naidoo, "Denoising autoencoder selforganizing map (dasom)," Neural Networks, vol. 105, pp. 112-131, 2018.

[17] L. Ren, Y. Sun, J. Cui, and L. Zhang, "Bearing remaining useful life prediction based on deep autoencoder and deep neural networks," Journal of Manufacturing Systems, vol. 48, pp. 71-77, 2018.

[18] Q. Xiang and X. Pang, "Improved denoising auto-encoders for image denoising," in 2018 11th International Congress on Image and Signal Processing, BioMedical Engineering and Informatics (CISP-BMEI), 2018, pp. 1-9. DOI: 10.1109/CISP-BMEI.2018.8633143.

[19] Z. Shi, Y. Feng, M. Zhao, and L. He, "A joint deep neural networks-based method for single nighttime rainy image enhancement," Neural Computing and Applications, pp. 1-14, 2019.

[20] S. Wang, Y. Zhang, D. Yang, and Z. Chen, "Ssim prediction for h. 265/hevc based on convolutional neural networks," in 2019 IEEE Visual Communications and Image Processing (VCIP), IEEE, 2019, pp. 1-4.

[21] T. Wang, X. Yang, K. Xu, S. Chen, Q. Zhang, and R. W. Lau, "Spatial attentive single-image deraining with a high quality real rain dataset," in Proceedings of the IEEE Conference on Computer Vision and Pattern Recognition, 2019, pp. 12 270-12279.

[22] S. Zhang, Y. Yao, J. Hu, Y. Zhao, S. Li, and J. Hu, "Deep autoencoder neural networks for short-term traffic congestion prediction of transportation networks," Sensors, vol. 19, no. 10, p. 2229, 2019.

[23] B. Ashwath, Indoor training set (its) [reside-standard], Oct. 2020. [Online]. Available: https : / / www . kaggle . com / balraj98 / indoor - training - set - its residestandard/metadata.

[24] A. Ceballos-Arroyo, S. Robles-Serrano, and G. S. Torres, "Remoción de lluvia en imágenes por medio de una arquitectura de autoencoder," Investigación e Innovación en Ingenierıas, pp. 140-167, 2020. 
[25] S. Lalmuanawma, J. Hussain, and L. Chhakchhuak, "Applications of machine learning and artificial intelligence for covid-19 (sars-cov-2) pandemic: A review," Chaos, Solitons \&6 Fractals, p. 110 059, 2020.

[26] R. Yamashita, M. Nishio, R. Do, and K. Togashi, Convolutional neural networks: An overview and application in radiology. insights imaging 9 (4), 611629 (2018). 